\title{
Flow Separation Control of Nacelle in Crosswind by Microsecond Pulsed Surface Dielectric Barrier Discharge Plasma Actuator
}

\section{Yuhao Jia ( $\sim$ Slone77777@163.com )}

Science and Technology on Plasma Dynamics Laboratory https://orcid.org/0000-0001-5842-2941

\section{Hua Liang}

Science and Technology on Plasma Dynamics Laboratory

\section{Qikun He}

Science and Technology on Plasma Dynamics Laboratory

\section{Zhi Su}

Science an Technology on Plasma Dynamics Laboratory

\section{Guoxing Song}

Science and Technology on Plasama Dynamics Laboratory

\section{Original research}

Keywords: plasma flow control, $\mu$ SDBD, nacelle inlet, crosswind condition

Posted Date: January 29th, 2021

DOl: https://doi.org/10.21203/rs.3.rs-157307/v1

License: (c) (1) This work is licensed under a Creative Commons Attribution 4.0 International License. Read Full License

Version of Record: A version of this preprint was published at Flow, Turbulence and Combustion on February 2nd, 2021. See the published version at https://doi.org/10.1007/s10494-021-00247-0. 


\section{Abstract}

Flow separation under crosswind conditions seriously jeopardizes the quality of the nacelle's flow field. In this paper, microsecond pulsed surface dielectric barrier discharge ( $\mu S D B D$ ) is used to suppress the flow separation and reduce the crosswind distortion of the nacelle. The flow structure induced by the $\mu S D B D$ is first explored by a high-speed schlieren system. The pressure waves composed of a cylindrical wave surrounding the electrodes and a flat wave at the top of the cylindrical one can be perceived, which indicates the fast gas heating produced by the $\mu S D B D$. A set of wind tunnel tests are then conducted to verify the ability of $\mu$ SDBD to suppress the nacelle flow separation and study the influence laws of pulse frequency, coverage area, and the actuator layout on the flow control effects. Results show that plasma actuation can not only improve the total pressure at the exit of the nacelle but also suppress the flow distortion caused by the crosswind. The best flow control effect can be achieved at the pulse frequency of $500 \mathrm{~Hz}$, with the value of sectional distortion coefficient reduced by $57.76 \%$ compared with the baseline condition. The flow control effect with the plasma actuator covering $120^{\circ}$ of the nacelle perimeter is better than that of $60^{\circ}$ and $180^{\circ}$ coverage, showing the highest flow control efficiency in the $120^{\circ}$ condition. The $\mu$ SDBD can improve mixing between the boundary layer and the main flow, enhancing the ability of the boundary layer to resist the adverse pressure gradient, which is beneficial to flow separation control.

\section{Introduction}

The large bypass ratio turbofan engines are widely used in civil airliners and military transport aircraft (Seddon et al. 1999), due to their advantages such as large thrust, low fuel consumption, and low noise (Joly et al. 2004). The nacelle is a critical component wrapping the large bypass ratio turbofan engine, which directly affects the performance and reliability of the engine thereby influences aircraft performance (Kang et al. 2011). Crosswind can induce flow separation at lateral positions of the nacelle inlet and distort the inlet airflow or even air crash due to the engine surge (Trapp et al. 2003; Vunnam et al. 2011).

To minimize the hazard brought by the crosswind, characteristics of the nacelle flow field and corresponding nacelle optimization have been extensively carried out (Hancock et al. 1969; Cesare et al. 2015). These works are mainly based on wind tunnel tests, bench crosswind tests, and flight testing platforms. A multiple curve modeling method was proposed to improve the aerodynamic performance of the turbofan engine nacelle (Qiang et al. 2013). Hall et al. (2012) conducted an experimental study of the $1 / 20$ nacelle scale model in the state of ground crosswind. The influence law of crosswind angle and ground clearance on the flow field characteristics was obtained and an obvious hysteresis effect during the separation and attachment of the airflow in the intake port was also found. The influence of crosswind speed and crosswind angle on the total inlet pressure distortion through the 1/11 nacelle scale model was also quantitatively analyzed (Murphy and Macmanus 2011). 
Numerical simulation of the flow field characteristics of the nacelle inlet is also a hot topic of research. The flow mechanism of the nacelle surface was qualitatively analyzed via three-dimensional CFD numerical simulation (Kamran 2013). In the design of the nacelle of the mixed-emission turbofan engine, CFD was used to simulate the shock waves, boundary layers, high-speed regions, and wake regions of the axisymmetric nacelle model (Tomita et al. 2011). By a set of software for numerically solving NavierStokes equations, the reliability of the numerical method and turbulence model was verified by comparison with experimental results (Tourrette et al. 2000). By simulating the crosswind problem of the nacelle, the ground vortex which exacerbated the distortion of the intake air under the crosswind condition was analyzed in detail (Luis and Roberto 2012).

However, few studies are devoting to suppress the flow separation of the nacelle inlet under crosswind conditions using flow control methods. A boundary layer control method was proposed (Hwang et al. 1994), which can delay the flow separation of the nacelle during the flight both at large angles of attack and in the cruise state. But there are also problems such as low efficiency and limited control range. In recent years, due to simple structure, fast response, large frequency bandwidth, and low energy consumption (Corke et al. 2009), surface dielectric barrier discharge (SDBD) has been widely used in airfoil (Little et al. 2010), laminar-to-turbulent flow transition control (Joussot et al. 2010), wing lift promotion (Greenblatt et al. 2008; Wei et al. 2020) and boundary layer acceleration (Im et al. 2010), and many other fields. Under the conditions of chord Reynolds numbers from $10^{4}$ to $10^{5}$ and freestream turbulence levels from 0.08 to $2.85 \%$, SDBD was applied for separation control on the low-pressureturbine blades. The obtained performance from plasma actuators was comparable to that of the vortex generators (Huang et al 2006). A single SDBD plasma actuator was used in a stationary, linear cascade blades tip clearance flow control at four Reynolds numbers: $5.3 \times 10^{4}, 6.9 \times 10^{4}, 8.7 \times 10^{4}$, and $1.03 \times$ $10^{4}$. The ability of SDBD to increases the flow turning in the tip region, and subsequently reduces the pressure loss due to the tip leakage vortex was verified (Van Ness et al. 2012). Control of the compressor tip leakage flow using SDBD plasma actuation was also studied by numerical and experimental methods under the flow velocity of $43 \mathrm{~m} / \mathrm{s}$. The optimal flow control effect in suppressing tip leakage flow was achieved with the axial plasma actuation (Zhang et al. 2019).

Compared with alternating current surface dielectric barrier discharge (AC-SDBD) and nanosecond pulsed surface dielectric barrier discharge (nSDBD), the pulse rise time of microsecond pulsed surface dielectric barrier discharge ( $\mu \mathrm{SDBD})$ is at a moderate level (several to tens of microseconds). Different from ACSDBD with limited flow control effects in high subsonic range (Li et al. 2006) and nSDBD with high power supply, weight and costs, $\mu S D B D$ can control the flow separation effectively in a wide range of freestream velocity with lower weight and costs. (Wei et al. 2020). The feasibility and efficacy of $\mu$ SDBD in the flight test with a scaled model of a certain type of amphibious plane were also verified (Su et al. 2018), which shows the potential of $\mu$ SDBD in flow separation control on nacelles.

In this paper, we used $\mu$ SDBD to improve the total pressure at the outlet of the nacelle and reduce the flow distortion due to the crosswind. The flow characteristics induced by $\mu$ SDBD at static atmosphere air is first investigated, and the formation of pressure waves and the corresponding mechanism of flow control 
are analyzed. Then, an integrated wind tunnel experiment system for flow suction and data acquisition is built with a DLR-F6 nacelle scale model, and the wind tunnel tests on flow separation control of nacelles by $\mu$ SDBD are conducted. The influence of the parameters including freestream velocity, engine suction speed, pulse frequency, and actuator layout and covering area, to the flow control is explored and the flow control mechanism is discussed. On this basis, an optimum plasma actuation method for nacelle flow separation control is obtained, which can help to improve the quality of the inlet flow field and broaden the flight envelope of the aircraft.

\section{Experimental Set-up And Tools}

\subsection{The nacelle inlet model and $\mu S D B D$ plasma actuation system}

The scale model of DLR-F6 nacelle for typical modern transport aircraft is used in the experiment (Vassberg et al. 2007). The nacelle model made of polyamide is 3D printed. As shown in Fig. 1 (b) the nacelle model is axisymmetric and the scaling factor is 20 . The lip diameter of the model is $D_{H L}=206$ $\mathrm{mm}$, and four groups of total pressure rakes are installed at an axial distance of $0.7 \mathrm{D}_{\mathrm{HL}}$ from the leading edge of the nacelle (Goldsmith and Seddon 1993). The total pressure rakes are radially distributed with the nacelle center as the origin, and there is a total of 28 measurement points. According to the principle of equal ring area (Wellborn et al. 1993), the radial distance from the measurement points of the total pressure rakes to the origin is $22.36 \mathrm{~mm}, 44.72 \mathrm{~mm}, 59.16 \mathrm{~mm}, 70.71 \mathrm{~mm}, 80.62 \mathrm{~mm}, 89.44 \mathrm{~mm}$, and $97.47 \mathrm{~mm}$. The four groups of total pressure rakes can be rotated by a rotation motor. In the experiment, the rotation range of the rotating motor is from $0^{\circ}$ to $90^{\circ}$ and the rotation angle is set to $10^{\circ}$. A total of 252 pressure taps are arranged and the total pressure value of each measuring point is the temporal average of each measurement point.

An asymmetric SDBD configuration (Zhou et al. 2010) is used in the experiment. The actuator consists of two electrodes (the exposed electrode and covered electrode) separated by a layer of dielectric (Kapton tape) without overlap, as shown in Fig. 2. The widths of exposed and covered electrodes are $3 \mathrm{~mm}$ and 5 $\mathrm{mm}$, respectively, and the thicknesses of both electrodes are $0.027 \mathrm{~mm}$. The dielectric layer with the relative permittivity of 3.4 has a thickness of $0.18 \mathrm{~mm}$. The installation of SDBD actuators in nacelle is divided into two layouts: streamwise direction and circumferential direction and Fig. 2 (b) is the installation diagram under two different layouts. The induced flow direction of the streamwise layout actuator is perpendicular to the incoming flow. The coverage area of the streamwise layout actuator is composed of multiple groups of single actuators. and the length of a single actuator is fixed to $36 \mathrm{~mm}$. The total length of the streamwise layout actuator is kept consistent with the circumferential layout actuator according to the experimental conditions (i.e. 60,120, and 180 degrees coverage area). The induced flow direction of the circumferential layout actuator coincides with the incoming flow, and the actuator is distributed along the leading edge of the inner ring of the lip. The total length of the two actuators is kept the same by changing the number of groups of the single streamwise actuator. 
The KGD-NSPS3U30F2 single output microsecond pulse power supply is adopted as a power source. The voltage amplitude ranges from 0 to $20 \mathrm{kV}$, while the pulse frequency is adjustable $(0-2 \mathrm{kHz})$ continuously. As shown in Fig. 3, the voltage waveform of $\mu$ SDBD generated by the discharge is a high-voltage pulse similar to a triangular wave and the peak-to-peak value of the pulse is $8 \mathrm{kV}$. The rising edge and falling edge are about 800ns, and the pulse width is about $1.3 \mu$ s. There is a current peak at the rising edge and the falling edge of the voltage. The peak value of the positive current of the discharge is $8 \mathrm{~A}$, and the peak value of the negative current can reach 17.9 A. Under a voltage pulse, the actuator discharges twice, corresponding to the rising edge and the falling edge of the voltage pulse and the second discharge current of $\mu S D B D$ is larger than the first time.

\subsection{Suction and pressure measurement devices}

Figure. 4 shows an integrated wind tunnel with suction airflow at the rear of the inlet and data acquisition devices. The experiment was conducted in the LSWT-1 unidirectional annular low-speed return wind tunnel with a turbulence level less than $0.2 \%$. The test section is $300 \mathrm{~cm}$ long with a cross-sectional size of $120 \mathrm{~cm} \times 100 \mathrm{~cm}$, and the stable wind speed ranges from 5 to $75 \mathrm{~m} / \mathrm{s}$. The nacelle model is fixed horizontally in the test section of the wind tunnel, and the crosswind conditions are simulated by adjusting the angle $\beta$ between the axis of the nacelle inlet and the incoming flow through the adjustment device. The tail of the inlet is connected to the suction system through a pipe with a constant crosssection. The suction machine is connected to the other end of the pipe whose suction volume can be adjusted by the control cabinet (range: $0 \sim 1.9829 \mathrm{~kg} / \mathrm{s}$ ) and is monitored in real-time with a pitot tube speedometer. The data acquisition system is used to obtain electrical and pressure parameters. The electrical parameter acquisition system is composed of a Tektronix P6015A voltage probe, a TCP0030A current probe and a DP04104 oscilloscope. The pressure acquisition system is composed of a pressure probe, the DSY-104 electronic pressure scanner and controller. The multi-channel pressure collecting valve of the DSY-104 is connected to the pressure probe through a hose with good airtightness, and the controller provides a time-dependent display and record of pressure data. The flow blockade area of experimental facilities is $1.42 \times 10^{-4} \mathrm{~m}^{3}$ and the blockade coefficient is $4.6 \%$.

\subsection{High-speed schlieren system}

The high-speed schlieren system is composed of a xenon lamp, a pair of convex lenses, a pair of plane mirrors, a pair of concave mirrors, a knife-edge, and a Phantom V2511 high-speed camera, see Fig. 5. The schlieren method is used to capture the characteristics of the flow field induced by $\mu$ SDBD plasma actuation in static atmospheric air. In this experiment, the ambient temperature is $27^{\circ} \mathrm{C}$ and the pressure is $10^{5} \mathrm{~Pa}$. The resolution of the high-speed camera is $512 \times 512$, with the frame rate ranging from 1000 to $50000 \mathrm{fps}$ and an exposure time of $0.98 \mu \mathrm{s}$. When performing a high-speed schlieren tset, the pulse width of the voltage during the discharge is set as $10 \mu \mathrm{s}$, and both the rising and falling edges are set as $800 \mathrm{~ns}$.

\section{Results And Discussion}




\subsection{Flow field characteristics induced by $\mu S D B D$ under the static atmosphere}

A sampling frequency of $50 \mathrm{kHz}$ corresponding to a sampling interval of $\Delta t=20 \mu \mathrm{s}$ is used in the experiment, and the schlieren images of $\mu$ SDBD plasma discharge with the peak-peak voltage of $V_{p-p}=12$ $\mathrm{kV}$ and pulse frequency of $f=1000 \mathrm{~Hz}$ were recorded with an exposure time of $0.98 \mu \mathrm{s}$. To make the image clearer and reduce background interference, the background normalization technique is used. That is, each schlieren image is subtracted by the pre-discharge image, and then divided by the average grayscale of the pre-discharge image to get a dimensionless relative intensity value of the pressure wave. In Fig. 6, the electrodes and dielectric layer of the plasma actuator are represented by rectangular color blocks, with an exposed electrode at the top, a dielectric layer in the middle, and a covered electrode at the bottom. A clear pressure wave can be observed during $\mu$ SDBD discharge. The pressure wave is cylindrical around the junction of the exposed and covered electrodes, and a flat wave is observed on the top of the cylindrical pressure wave. This is consistent with the typical topology of pressure waves caused by nSDBD mentioned in (Zhang et al. 2019; Nicolas et al. 2012), which is a cylindrical wave surrounding the electrode and a flat wave leaving the surface. The cylindrical part of the pressure wave is caused by the energy released from the edge of the exposed electrode, and the generation of flat wave is originated from the energy deposition during streamer propagation stage (Starikovskii et al. 2009).

After the discharge begins ( $t=20 \mu \mathrm{s})$, the cylindrical pressure wave with a radius of $r=7.04 \mathrm{~mm}$ is formed. Over time, the pressure wave expands continuously, as well as the planar wave. When $t=60 \mu \mathrm{s}$, the intensity of the cylindrical pressure waves on both sides gradually weakens, while the intensity of pressure waves in and around the planar area remains essentially unchanged. A second pressure wave with low brightness can be seen at $t=120 \mu \mathrm{s}$. At the beginning of the discharge, a pressure wave will be generated on both the rising and falling edges of the microsecond pulse voltage, but the latter has a weak pressure wave strength. Benard et al. (2012) also pointed out that a pressure wave is generated on the rising and falling edges of the voltage. The intensity of the pressure wave is closely related to the voltage amplitude and the pulse rise time, and the positive pulse discharge shows a higher intensity. Only one pressure wave will be observed when the amplitude is low and after the voltage increases to a certain value, two pressure waves will be found and the flat wave can also be observed. When $t=120 \mu \mathrm{s}$, the range of the cylindrical pressure wave is further expanded to $r=41.84 \mathrm{~mm}$. Figure 7 records the radius and propagation speeds of the cylindrical pressure wave induced by $\mu$ SDBD. In our experiment, the velocity resolution of the high-speed schlieren system is $20.6 \mathrm{~m} / \mathrm{s}$. The propagation speed of the pressure wave is maintained at around $350 \mathrm{~m} / \mathrm{s}$ (see Fig. 7), the value approximately equals to the local sound velocity, the phenomenon is in reasonable agreement with the results in (Zhang et al. 2019; Xie et al. 2019). The results indicate that $\mu$ SDBD, nSDBD, and microsecond surface arc discharge ( $\mu S A D)$ have similar pressure wave structures and propagation speeds. Moreover, a thermal mass of $4 \mathrm{~mm} \times 4 \mathrm{~mm}$ can be observed above the junction of the exposed and covered electrodes of the actuator, and Zhu et al. (2013) pointed out that the generation of cylindrical pressure waves is due to fast energy release.

From the point of fluid transport, the thermal mass develops from the edge of the electrode, so the cylindrical wave develops around the edge of the electrode. This phenomenon has also been verified by 
the numerical simulation of (Unfer and Boeuf 2010). In the current research, the pulse width of the voltage during the discharge is $10 \mu \mathrm{s}$, both the rising and falling edges are $800 \mathrm{~ns}$. This shows the energy release in a microsecond time scale, which results in a dramatic rising in the temperature and realizes "fast heating". The heat release leads to a rapid increase in local pressure because the time scale of density variations is much longer than temperature rising. When the local pressure exceeds a certain threshold, a pressure wave is generated due to gas expansion from the surface, exerting a short-term impact on the fluid, which is similar to the flow control mechanism of nSDBD actuation (Roupassov et al. 2008). Some researchers have claimed the main factor of an $\mathrm{nSDBD}$ to be the pressure wave due to rapid gas heating in the pulsed discharges (Zheng et al. 2014). Other researchers have claimed that two lumps of heated air were produced by the nSDBD actuation: one propagated along the boundary between the main and separated flows. The other propagated along the surface more slowly than the main flow. This heated air might excite the shear-layer instability, and the induced vortex shedding could result in momentum transfer and entrainment enhancement to the separated flow through the separated shear layer (Komuro et al. 2017; Komuro et al 2018).

\subsection{Characteristics of nacelle inlet under crosswind condition}

When the nacelle inlet is too close to the ground, the ground effect will induce the strong ground suction vortex, causing flow field distortion (Brix et al. 2000). To avoid the influence of the ground effect on the experimental results, the vertical distance between the nacelle inlet and the wind tunnel wall is set to be larger than $2 \mathrm{D}_{\mathrm{H}}$. A high-performance inlet must ensure a small total pressure loss and an evenly distributed flow filed at the exit (Seddon et al. 1985). Total pressure recovery coefficient $P R$ is used to characterize the loss of the intake flow (Harrison et al. 2013), which is defined as the ratio of the average total pressure $P_{f}$ (spatial average value of 252 measurement points) at the nacelle exit to the total pressure $P_{0}$ of the undisturbed cross-section flow, ie:

$$
P R=\frac{P_{f}}{P_{0}}
$$

The flow field distortion is estimated by $D C(60)$, a parameter to measure the quality of the inlet flow field, which is given as:

$$
D C(60)=\frac{P_{f}-P_{\infty}}{\frac{1}{2} \rho U_{\infty}^{2}}
$$

Where $P_{60}$ is the worst average 60 degree sector total pressure, see Fig. 8 . At $27^{\circ} \mathrm{C}$, the density of air $\rho$ is $1.177 \mathrm{~kg} / \mathrm{m}^{3}$ and $U_{\infty}$ is the velocity of the incoming flow.

The flow coefficient $\varphi$ is usually used to characterize the flow capacity of the inlet. Under a certain flight Mach number, the flow coefficient is the ratio of the mass flow of air actually entering the inlet to the 
mass flow of air sucked in without decelerating boost or accelerating decompression at the same Mach number, defined as:

$$
\varphi=\frac{\rho_{\infty} U_{\infty} A_{\infty}}{\rho_{\infty} U_{\infty} A_{c}}=\frac{A_{\infty}}{A_{c}}
$$

Where $A_{\infty}$ is the free flow tube area for far-field incoming flow. $A_{c}$ is the inlet flow capture area (i.e. the projected area of the leading edge of the nacelle lip in a plane perpendicular to the direction of incoming flow). $U_{\infty}$ is the far-field incoming flow velocity. $\rho_{\infty}$ is the far-field incoming air density.

From the conservation of fluid mass:

$$
\frac{U_{i}}{U_{\infty}}=\frac{\rho_{\infty}}{\rho_{i}} \frac{A_{\infty}}{A_{i}}
$$

In the experiment, the incoming flow velocity is less than $0.3 \mathrm{Ma}$. The compressibility is not to be considered, so the incoming flow density remains unchanged, i.e.

$$
\varphi=\frac{U_{i}}{U_{\infty}}
$$

To satisfy the requirements of safe operation, the inlet in the ground state should achieve a high quality of air-intake when the flow speed of the wind tunnel is larger than $10.29 \mathrm{~m} / \mathrm{s}$ (20 knots) (Colin et al. 2007). To simulate the typical state in the take-off phase, this paper selects the flow speed of the wind tunnel of $U_{\infty}=15.42 \mathrm{~m} / \mathrm{s}$ (30 knots), and the effect of crosswind angle on the flow field distortion was studied when the engine suction speed $U_{i}=20 \mathrm{~m} / \mathrm{s}$.

As shown in Fig. 9, when the angle of crosswind is small $\left(\beta=10^{\circ}\right), P R$ along the whole cross-section is commonly high. A low-pressure area is mainly located near the wall and the lower right corner. The total pressure loss here is caused by the viscosity loss of the near-wall flow and the slight flow separation resulted from the crosswind. When $\beta=14^{\circ}$, low-pressure area is much obvious on the windward side, the quality of the outlet flow field gets worse. When $\beta$ increases to $18^{\circ}, P R$ in the low-pressure area becomes less than 0.9975 . As $\beta$ increases, $P R$ further decreases, and the degree of flow separation also exacerbates. At a large crosswind angle of $\beta=45^{\circ}$, the low-pressure area $P R$ is reduced to below 0.993 , and the flow separation range expands to $50 \%$ of the cross-section. This shows that the total pressure distortion at the inlet exit is severe and the quality of the outlet flow field deteriorates sharply. In general, as the crosswind angle increases, the separation flow area begins to expand, and its influence range also continues to expand, resulting in a reduction in the $P R$ and a large low-pressure area, which ultimately leads to intake distortion increase. 
Based on the analysis above, the flow separation is obviously intensified at $\beta=14^{\circ}$, and the range of lowpressure area is large, so $\beta=14^{\circ}$ is a critical value for the deterioration of the flow field quality. To study the influence of the suction velocity $U_{i}$ on the flow field characteristics of the nacelle inlet, $\beta$ is fixed at $14^{\circ}$, and the $D C(60)$ are tested at three different speeds: $U_{\infty}=15.42 \mathrm{~m} / \mathrm{s}$ (30 knots), $25.70 \mathrm{~m} / \mathrm{s}$ (50 knots), $35.98 \mathrm{~m} / \mathrm{s}$ (70 knots). The profiles of the $D C(60)$ as $U_{i}$ is shown in Fig. 10 . When $U_{\infty}=15.42 \mathrm{~m} / \mathrm{s}, 25.70$ $\mathrm{m} / \mathrm{s}$ and $35.98 \mathrm{~m} / \mathrm{s}, D C(60)$ quickly decreases to the value below 0.3 at $U_{i}=19.96 \mathrm{~m} / \mathrm{s}, 19.06 \mathrm{~m} / \mathrm{s}$ and $18.30 \mathrm{~m} / \mathrm{s}$ respectively, and then decreases continuously, eventually reaching 0 . The flow coefficients $\varphi$ of these turning points are $1.29,0.74$, and 0.51 , and the range of $\varphi(0.5 \sim 1.5)$ can reflect the typical crosswind conditions of the nacelle inlet from climb to level flight.

The phenomenon above can also be verified at $\beta=18^{\circ}$ and $22^{\circ}$. Flow separation appears when the crosswind angle is relatively small $\left(\beta=14^{\circ} \sim 22^{\circ}\right)$. $P R$ decreases mean the total pressure distortion degree increasing and the quality of the flow field worsening. The flow separation inside the inlet under crosswind conditions is the main reason for nacelle inlet distortion, and inlet distortion will suddenly increase with increasing crosswind speed. Especially when the speed is high (i.e. $U_{\infty}=35.98 \mathrm{~m} / \mathrm{s}$ ), the crosswind separation range is wider, and the total pressure loss is also greater. Next, we will use $\mu$ SDBD to explore the plasma flow control technology on increasing $P R$ under crosswind conditions, reducing the crosswind separation regions, and improving the quality of the flow field.

\subsection{Flow control effects of streamwise plasma actuation}

To verify the flow separation control effect of the nacelle inlet under crosswind conditions by $\mu$ SDBD plasma actuation, the experiment was carried out in a small angle crosswind range $\left(\beta=14^{\circ}, 18^{\circ}\right)$ to avoid the influence of reduced circulation capacity of the nacelle on the separation flow field. The layout of $\mu S D B D$ actuator is streamwise.

\subsubsection{Influence of pulse frequency on the flow control effect}

Previous studies have shown that the unsteady plasma actuation can effectively improve the flow control effect with lower energy consumption accounting for the coupling of the flow field instability with shedding vortex frequency (Greenblatt et al. 2008). The effect of high-voltage pulse frequency on the flow control was studied in this section. The flow speed of the wind tunnel and the suction speed is fixed at $U_{\infty}$ $=25.70 \mathrm{~m} / \mathrm{s}$ and $U_{i}=25.74 \mathrm{~m} / \mathrm{s}$ respectively to simulate the work states during the climbing stage of aircraft (flow coefficient $\varphi$ is close to 1 ). The voltage waveform with the peak-peak value $V_{p-p}=8 \mathrm{kV}$, pulse width of $10 \mu \mathrm{s}$ and $800 \mathrm{~ns}$ rising and falling edges was applied to the actuator. The experiment was conducted under three actuation frequencies $f=500 \mathrm{~Hz}, 1000 \mathrm{~Hz}$, and $2000 \mathrm{~Hz}$. Obtained $P R$ and $D C(60)$ at the air-intake exit are given in Tab. 1.

Tab. 1 The air-intake exit $P R$ and $D C(60)$ 


\begin{tabular}{|c|c|c|c|c|c|c|}
\hline$\beta \rrbracket^{\circ} \nabla$ & 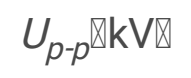 & $f \otimes H z \bigotimes$ & $P R$ & $\triangle P R$ & $D C(60)$ & $\Delta D C(60)$ \\
\hline 14 & \multirow[t]{2}{*}{0} & \multirow[t]{2}{*}{0} & 0.999024 & \multirow[t]{2}{*}{-0.000925} & 0.146748 & \multirow[t]{2}{*}{0.882576} \\
\hline 18 & & & 0.998099 & & 1.029324 & \\
\hline 14 & \multirow[t]{2}{*}{8} & \multirow[t]{2}{*}{500} & 0.999103 & \multirow[t]{2}{*}{-0.000275} & 0.115946 & \multirow[t]{2}{*}{0.372833} \\
\hline 18 & & & 0.998828 & & 0.488779 & \\
\hline 14 & \multirow[t]{2}{*}{8} & \multirow[t]{2}{*}{1000} & 0.999098 & \multirow[t]{2}{*}{-0.000309} & 0.119526 & \multirow[t]{2}{*}{0.402375} \\
\hline 18 & & & 0.998789 & & 0.521901 & \\
\hline 14 & \multirow[t]{2}{*}{8} & \multirow[t]{2}{*}{2000} & 0.999103 & \multirow[t]{2}{*}{-0.00035} & 0.116946 & \multirow[t]{2}{*}{0.454253} \\
\hline 18 & & & 0.998753 & & 0.571199 & \\
\hline
\end{tabular}

The $P R$ decreases with the crosswind angle $\beta$, while the $D C(60)$ increases with $\beta$. When the crosswind angle increases by $4^{\circ}$ without actuation, the $P R$ at nacelle exit decreases 0.000925 . With $\mu$ SDBD plasma actuation, the $P R$ of the air-intake exit is reduced by 0.00275 at $f=500 \mathrm{~Hz}$. As the actuation frequency increases to $1000 \mathrm{~Hz}$ and $2000 \mathrm{~Hz}$, the $P R$ of the air-intake exit decreases by 0.000309 and 0.00035 respectively, which are lower than that of without actuation. It shows that $\mu$ SDBD plasma actuation can improve the effect of $P R$ reduction when the crosswind angle becomes larger. For example, $P R$ can be significantly improved by $70.27 \%$ when $f=500 \mathrm{~Hz}$. Without actuation, $D C(60)$ changed from 0.146748 to 1.029324 , increased by 0.882576 when the crosswind angle becomes $18^{\circ}$. After applying $\mu S D B D$ plasma actuation with $f=500 \mathrm{~Hz}, 1000 \mathrm{~Hz}$, and $2000 \mathrm{~Hz}, D C(60)$ only increases by $0.372833,0.402375$, and 0.454253 , which were $57.76 \%, 54.20 \%$, and $48.53 \%$ lower than that of without actuation (0.882576). This indicates that plasma actuation can reduce the distortion of the flow field when the crosswind angle increases effectively.

To further characterize the effect of plasma actuation on the flow separation control and reducing the deterioration of the quality of the flow field at the air-intake exit, Figure 11 shows the distribution of the $P R$ under different frequencies. Plasma actuation can reduce the range of the low-pressure area of the airintake exit and suppress the flow separation at different crosswind angles. At $\beta=14^{\circ}$, the plasma actuation can not only reduce the size of the low-pressure area but also change the position of flow separation. The low-pressure area in Fig. 11(a) is located in the lower right corner, and its circumferential range is about $45^{\circ}$. With plasma actuation of $f=500 \mathrm{~Hz}$, the low-pressure area shifted to the upper right and the circumferential range is reduced to approximately $25^{\circ}$. However, as the actuation frequency continues to increase, the location and range of the low-pressure area can hardly change, indicating that the $\mu$ SDBD plasma actuation saturated at $V_{p-p}=8 \mathrm{kV}$ and increasing the frequency can no longer improve the flow control effect. At $\beta=18^{\circ}$, the low-pressure area of the baseline flow field is more obvious, whose range is about $145^{\circ}$ and the $P R$ value is relatively low (0.996). Similarly, with plasma actuation of $f=500$ 
$\mathrm{Hz}$, the low-pressure area is significantly reduced and the range is reduced to $45^{\circ}$. When the frequency increase to $f=1000 \mathrm{~Hz}$ and $2000 \mathrm{~Hz}$, although the range of the low-pressure area can also be reduced to $60^{\circ}$ and $66^{\circ}$, the flow control effect is not as good as that at $f=500 \mathrm{~Hz}$. Analysis reason: It may be related to the structure of the flow field. Under crosswind conditions, the flow may be accompanied by the generation, development and shedding of vortices. As in airfoil flow control, there is an optimal coupling frequency for ns-DBDPA, Synthetic Jet Actuator, and High-frequency micro-vortex-generator. Thus, $\mu S D B D$ also has an optimal coupling frequency in nacelle separation control. Because the experiment was carried out at a low speed, the main frequency of the vortex is relatively low. Besides, the actuation characteristics have a certain relationship with the frequency. When the actuation frequency is greater than the optimal frequency, the flow control effect will be worse (Gursul et al. 2007 and Wei et al. 2020).

The $\mu$ SDBD plasma actuation can improve the effect of $P R$ reduction when the $\beta$ becomes larger. Besides, it can suppress the flow separation of the inlet and the distortion of the airflow at the nacelle exit under crosswind conditions. The flow control effect is best under the frequency $f=500 \mathrm{~Hz}$ and increasing the frequency to $1000 \mathrm{~Hz}$ and $2000 \mathrm{~Hz}$ cannot improve the flow control effect. It has to be noted that, the discussion on the frequency is based on this experiment condition. When under the actual flight conditions, the optimal value of the actuation frequency would be influenced by the factors such as crosswind speed, angle, and nacelle size. After applying the $\mu$ SDBD plasma actuation, the pulse energy is concentratedly released in a very short time, resulting in the instantaneous heating of the surrounding gas. The fast heat gas charges the surface of the dielectric layer and enhances the mixing of mass and momentum between the boundary layer and the main flow, injecting more energy into the bottom of the boundary layer. So that the separated boundary layer reattaches. Thus, the flow control effect is achieved and the power consumption is reduced at the same time. The $\mu$ SDBD plasma actuation can reduce the separation area effectively, resulting in improvement of $P R$ and reduction of distortion of the airflow at the air-intake exit.

\subsubsection{Energy optimization of streamwise plasma flow control}

Limited by the power rating, to improve the flow control efficiency of $\mu$ SDBD, it is important to optimize the actuator coverage area to achieve good flow control effects under the lowest power consumption. The analysis above indicated that when the crosswind angle is small, the low-pressure area of the airintake exit is about $60^{\circ} \sim 120^{\circ}$. While the arrangement range of the streamwise layout is $180^{\circ}$, the difference may cause energy waste. To further explore the more energy-efficient configuration, the groups of single actuators are set to 3,6 and 9 to control the actuator coverage area to $60^{\circ}, 120^{\circ}$, and $180^{\circ}$ under the condition of $U_{\infty}=25.70 \mathrm{~m} / \mathrm{s}, \beta=14^{\circ}, f=500 \mathrm{~Hz}, V_{p-p}=8 \mathrm{kV}$. The center point of the coverage area is the midpoint of the half-circle on the windward side.

Figure 12 shows $P R$ of the air-intake exit with the streamwise layout of $60^{\circ}, 120^{\circ}$, and $180^{\circ}$ coverage. The $P R$ of $120^{\circ}$ coverage is higher than the other two, however, with the increase of $U_{i}$, the difference among each other decrease and finally was relatively close. The distribution of $P R$ under different actuator coverage is shown in Fig. 13. After the actuation is applied, when $U_{i}=13.54 \mathrm{~m} / \mathrm{s}$ and the coverage is $60^{\circ}$ 
and $180^{\circ}$, the low-pressure areas are significantly larger than the coverage of $120^{\circ}$. When $U_{i}$ reaches $16.69 \mathrm{~m} / \mathrm{s}$, the $120^{\circ}$ coverage can suppress the flow separation inside the inlet and make the lowpressure area disappears. While with $60^{\circ}$ and $180^{\circ}$ coverage can only achieve the same effect when $U_{i}$ reaches $19.84 \mathrm{~m} / \mathrm{s}$. According to the obtained data (see Fig. 14), the $\mu$ SDBD actuation discharge energy of different coverage areas is calculated by integrating the product of current and voltage. The result of $60^{\circ}, 120^{\circ}$, and $180^{\circ}$ coverage are respectively $0.732 \mathrm{~mJ}, 1.527 \mathrm{~mJ}$, and $2.024 \mathrm{~mJ}$, indicating that the $120^{\circ}$ coverage $\mu$ SDBD has better control effect and low energy consumption.

In general, when the streamwise layout $\mu$ SDBD coverage is small, increasing the group of single actuators can expand the action range of the plasma aerodynamic actuation and make it more effectively perform flow control under the same actuation conditions. While the coverage reaches a certain threshold, continuing to expand the coverage area has little effect on improving the flow control effect. And a voltage drop will occur due to the power-supply limitation. Also, the range of the separation area is about $120^{\circ}$, which is as large as the control area of $120^{\circ}$ coverage $\mu$ SDBD. Compared with $180^{\circ}$ coverage $\mu S D B D, 120^{\circ}$ coverage $\mu$ SDBD can better concentrate the power of the power-supply to control the separation area. Therefore, the $120^{\circ}$ coverage $\mu$ SDBD gained low energy consumption for all given conditions with the scalable structure and operation, which is ideal for controlling nacelle flow separation.

\subsection{Comparative research on flow control effect of streamwise and circumferential layout}

An experiment (Jolibois et al. 2008) revealed that the layout of SDBD actuators could achieve different flow control effects by changing the direction of actuation. To explore the layout's influence on flow separation, the actuator was arranged streamwise and circumferentially in this work. Experiments under different crosswind speeds and different suction speeds were carried out. The voltage with $V_{p-p}=8 \mathrm{kV}$ and the optimum frequency $f=500 \mathrm{~Hz}$ is used, and crosswind angle $\beta=14^{\circ}$. The coverage area of the actuator was set to $120^{\circ}$.

The air-intake exit $P R$ and $D C(60)$ under different actuator layouts are shown in Figs. 15 and 16. When $U_{\infty}$ $=25.70 \mathrm{~m} / \mathrm{s}$, there is little difference for air-intake exit $P R$ between two layouts. The control effect decreases with the suction speed. Either $U_{\infty}=25.70 \mathrm{~m} / \mathrm{s}$ or $U_{\infty}=35.98 \mathrm{~m} / \mathrm{s}, D C(60)$ of the streamwise layout is significantly smaller than that of the circumferential layout. When $U_{\infty}$ reaches $35.98 \mathrm{~m} / \mathrm{s}$, the streamwise layout reduces $D C(60)$ to $1.4076,0.4474,0.1408$, and 0.1159 at the $U_{i}=13.54 \mathrm{~m} / \mathrm{s}, 16.69$ $\mathrm{m} / \mathrm{s}, 19.84 \mathrm{~m} / \mathrm{s}$, and $22.98 \mathrm{~m} / \mathrm{s}$. Compared with the circumferential layout actuation, $D C(60)$ is reduced by $19.45 \%, 30.28 \%, 30.01 \%$, and $41.68 \%$, indicating that the former has a better control effect.

To better reflect two layouts' difference in flow control, Figure 17 and Figure 18 respectively show the total pressure distribution at air-intake exit under different actuator layouts when $U_{\infty}=25.70 \mathrm{~m} / \mathrm{s}$ and $U_{\infty}=$ 
$35.98 \mathrm{~m} / \mathrm{s}$. When $U_{\infty}=25.70 \mathrm{~m} / \mathrm{s}$ and $U_{i}$ reaches $19.84 \mathrm{~m} / \mathrm{s}$ and $22.98 \mathrm{~m} / \mathrm{s}$, the low-pressure area is obviously reduced after applying streamwise layout. The flow separation generally disappears and the total pressure distortion is also significantly reduced. When using the circumferential layout, the lowpressure area is larger than that of the streamwise layout and the $P R$ of the high-pressure area is lower. After the $U_{\infty}$ increased to $35.98 \mathrm{~m} / \mathrm{s}$, the low-pressure area of the streamwise layout is smaller than the circumferential layout. The results indicate that the control effect of the streamwise layout is better than that of the circumferential layout, especially in low crosswind speed.

In general, there are two ways for plasma aerodynamic actuation to control the boundary layer: one is to increase the flow velocity in the boundary layer The other is to enhance the energy exchange of the boundary layer and the mainstream (Porter et al. 2007). The actuation position is crucial to suppress the flow separation Circumferential layout can induce gas flow to accelerated. Both are injecting energy into the boundary layer through discharges, which causes disturbance to the near-wall flows, promotes the mixing between the low-energy flow and the mainstream high-energy flow in the boundary layer and enhances the ability of the boundary layer to resist the backpressure gradient. The streamwise layout wraps the entire nacelle front edge to the vicinity of the separation point. Its actuation area is larger, while the circumferential layout is arranged in front of the separation point and it is not clear whether this is its optimum actuation position. In order to more effectively suppress the flow separation, it is necessary to conduct a deeper study on the position of the $\mu$ SDBD.

\section{Conclusions}

This paper explores the topological structure of pressure waves induced by $\mu$ SDBD as well as the mechanism of impact disturbance to control flow separation. The pressure wave consists of a cylindrical wave surrounding the electrode and a flat wave at the top of the cylindrical one. The pulse energy of $\mu S D B D$ is concentratedly released at the microsecond level, generating a thermal mass.

The basic flow field characteristics of the nacelle inlet under crosswind conditions are obtained. The crosswind angle and velocity seriously affect the quality of the air-intake exit flow field. The greater the angle and velocity are, the lower $P R$ of the exit cross-section is, the more severe the flow separation will be, causing a greater total pressure distortion.

A series of wind tunnel experiments are carried out on the nacelle inlet under crosswind conditions using a $\mu S D B D$. The influence law and action mechanism of different actuation frequency, actuator layout, and optimum layout of the actuator on the flow control effect are explored. $\mu$ SDBD plasma actuation $\left(V_{p-p}=\right.$ $8 \mathrm{kV}, f=500 \mathrm{~Hz}, 1000 \mathrm{~Hz}$, and $2000 \mathrm{~Hz}$ ) can suppress the flow separation of the inlet and the distortion of the airflow caused by the crosswind conditions. When $f=500 \mathrm{~Hz}$, the control effect of the $\mu$ SDBD actuation is better, reducing the value of $D C(60)$ by $57.76 \%$ compared with no actuation. To improve the efficiency of the $\mu$ SDBD plasma actuator, the layout of the streamwise layout $\mu$ SDBD actuator is 
optimized. It was found that when the streamwise layout $\mu$ SDBD coverage is $120^{\circ}$, its control effect is better than $60^{\circ}$ and $180^{\circ}$. And the energy consumption of $120^{\circ}$ coverage actuator is also lower for all given conditions with the scalable structure and operation. The streamwise layout $\mu$ SDBD plasma actuation greatly reduces the total pressure distortion. Compared with circumferential layout actuation, the low-pressure area is significantly smaller. $D C(60)$ is reduced by $19.45 \%, 30.28 \%, 30.01 \%$ and $41.68 \%$ at $U_{i}=13.54 \mathrm{~m} / \mathrm{s}, 16.69 \mathrm{~m} / \mathrm{s}, 19.84 \mathrm{~m} / \mathrm{s}$ and $22.98 \mathrm{~m} / \mathrm{s}$ The streamwise layout actuator has better ability to control flow separation.

More detailed and comprehensive actuator optimized layout design, as well as the mechanisms of $\mu S D B D$ actuation on nacelle flow control, will be presented in further works based on more detailed works including numerical simulation and experiments.

\section{Declarations}

\section{Acknowledgements}

The authors acknowledge funding by the Major Program of the National Natural Science Foundation of China (Grant agreement No.51790511), the National Natural Science Foundation of China (Grant agreement No.91941105,91941301). Anonymous referees are thankfully acknowledge for insightful comments on the first draft of this manuscript.

\section{Ethics declarations}

\section{Conflict of interest}

The authors report no conflict of interest.

\section{References}

1. Seddon, J., Goldsmith, E.: Intake Aerodynamics. United Kingdom: Blackwell (1999).

2. Joly, R.B., Ogaji, S.O.T., Singh, R., et al.: Gas-turbine diagnostics using artificial neural-networks for a high bypass ratio military turbofan engine. Applied Energy 78(4):397-418 (2004).

3. Kang, C.H., Hsu, W.C., Lee, E.K., et al.: Dynamic analysis of gear-rotor system with viscoelastic supports under residual shaft bow effect. Mechanism and Machine Theory 46(3): 264-275 (2011).

4. Trapp,, Oliveira, G.: Aircraft thrust reverser cascade configuration evaluation through CFD. 41st Aerospace Sciences Meeting and Exhibit. 723 (2003).

5. Vunnam „Hoover, R.: Modeling of inlet distortion using a combined turbofan and nacelle inlet model during crosswind and low speed forward operation. Turbo Expo: Power for Land, Sea, and Air. 54617, 371-380 (2011).

6. Hancock, J.P., Hinson, B.: Inlet development the L-500. AIAA 69-488, 1969 (1969). 
7. Cesare, A., Hall, Thomas,, Hynes.: Measurements of Intake Separation Hysteresis in a Model Fan and Nacelle Rig. journal of propulsion \& power, 2012, 22(4):872-879 (2012).

8. Qiang, X.H., Wang, Z.H., Liu, Z.W., Zhang, X.B., Zhang, J.W.: Design Method Research of TurbofanEngine Nacelle. Machinery Design \& Manufacture 2013(11):23-25 (2013).

9. Hall, C.A., Hynes, T.P.: Measurements of Intake Separation Hysteresis in a Model Fan and Nacelle Rig. Journal of Propulsion \& Power 22(4):872-879 (2012).

10. Murphy,P., Macmanus, D.G.: Ground vortex aerody-namics under crosswind conditions. Experiments in Fluids 50(1): 109-124 (2011).

11. Kamran,: Viscous flow past a nacelle isolated and in proximity of a flat plate.AIAA Journal 2013:919ه 927 (2013).

12. Tomita, J.T., Bringhenti, C., Barbosa, J.R., et al.: Nacelle design for mixed turbofan engines.Turbo Expo: Power for Land, Sea, and Air (2006).

13. Tourrette L.: Navier-Stokes Simulations of Air-Intakes in Crosswind Using Local Preconditioning. AIAA Journal 2002-2739, 2000 (2002).

14. Luis, G.T. and Girardi, R.: Evaluation of Engine Inlet Vortices Using CFD. AIAA 2012-1200 (2012).

15. Hwang, D., Boldman, D., Hughes, C.: Flow analysis for the nacelle of an advanced ducted propeller at high angle of attack and at cruise with boundary layer control. 32nd Aerospace Sciences Meeting and Exhibit: (1994).

16. Corke, T.C., Enloe, C.L., Wilkinson, S.P.: Dielectric Barrier Discharge Plasma Actuators for Flow Control. Annual Review of Fluid Mechanics 42(1):505-529 (2009).

17. Little, J., Nishihara, M., Adamovich, I., et al.: High-lift airfoil trailing edge separation control using a single dielectric barrier discharge plasma actuator. Experiments in fluids. 48(3): 521-537 (2010).

18. Feng, L.H., Jukes, T.N., Choi, K.S., et al.: Flow control over a NACA 0012 airfoil using dielectric-barrierdischarge plasma actuator with a Gurney flap. Experiments in fluids. 52(6): 1533-1546 (2012).

19. Joussot, R., Hong, D., Weber-Rozenbaum, R., et al.: Modification of the laminar-to-turbulent transition on a flat plate using DBD plasma actuator. 5th Flow Control Conference. 4708 (2010).

20. Greenblatt, D., Kastantin, Y., Nayeri, C.N., et al.: Delta-Wing Flow Control Using Dielectric Barrier Discharge Actuators. AIAA Journal. 46(6):1554-1560 (2008).

21. Wei, B., Wu, Y., Liang, H., et al.: Flow control on a high-lift wing with microsecond pulsed surface dielectric barrier discharge actuator. Aerospace Science and Technology. 96, 105584 (2020).

22. Im, S., Do, H., Cappelli, M.A.: Dielectric barrier discharge control of a turbulent boundary layer in a supersonic flow. Applied Physics Letters. 97(4):605 (2010).

23. Huang, J., Corke, T.C., Thomas, F.O.: Plasma Actuators for Separation Control of Low-pressure Turbine Blades. AIAA Journal 44(1): 51-57 (2006).

24. Van Ness, D.K., Corke, T.C., Morris, S.C.: Plasma actuator blade tip clearance flow control in a linear turbine cascade. Journal of Propulsion and Power. 28(3): 504-516 (2012). 
25. Zhang, H., Wu, Y., Li, Y., et al.: Control of compressor tip leakage flow using plasma actuation. Aerospace Science and Technology. 86: 244-255 (2019).

26. Li, Y.H., Wu, Y., Song, H.M., et al.: Preliminary Experimental Investigation of Atmosphere Plasma Flow Control Technology. Journal of Air Force Engineering University (Natural Science Edition) 3 (2006)..

27. Zhao, Y., Li, Y.H., Liang, H., et al.: Phenomenological modeling of nanosecond pulsed surface dielectric barrier discharge plasma actuation for flow control. Acta Physica Sinica 64(1):15101015101 (2015).

28. Wu, Y.: Nanosecond pulsed discharge plasma actuation: characteristics and flow control performance. 45th AIAA Plasmadynamics and Lasers Conference. 2118 (2014).

29. Goldsmith, E.L., Seddon, J.: Practical intake aerodynamic design. Amer Inst of Aeronautics \&. (1993).

30. Wellborn, S.R., Okiishi, T.H., Reichert, B.A.: A study of the compressible flow through a diffusing Sduct. NASA Technical Memorandum 106411 (1993).

31. Zhou, B., Wang, X.J , Sun, C.X.: Effect of electrode structure on the parameters of dielectric barrier discharge. High Voltage Apparatus 46(4):31-34+39 (2010).

32. Zhang, Huang, B.D., Luo, Z.B., Che, X.K., Yan, P., Shao, T.: Atmospheric-pressure pulsed plasma actuators for flow control: shock wave and vortex characteristics. Plasma Sources Science and Technology 28: 064001 (2019).

33. Nicolas, B., Nourredine, Z., Alain, C., Julien, S., Eric, M.: Optical visualization and electrical characterization of fast-rising pulsed dielectric barrier discharge for airflow control applications. Journal of Applied Physics 111: 033303 (2012).

34. Starikovskii, A.Y., Nikipelov, A.A., Nudnova, M.M., et al.: SDBD plasma actuator with nanosecond pulse-periodic discharge. Plasma Sources Science and Technology 18(3): 034015 (2009).

35. Xie, L.K., Liang, H., Zhao, G., et al.: Characteristics of pulsed surface arc discharge actuator and its verification of flow control over a Delta wing. Sensors and Actuators A: Physical 297: 111550 (2019).

36. Zhu, Y., Wu, Y., Cui, W., et al.: Modelling of plasma aerodynamic actuation driven by nanosecond SDBD discharge. Journal of Physics D: Applied Physics 46(35): 355205 (2013).

37. Unfer, T., Boeuf, J.P.: Modeling and comparison of sinusoidal and nanosecond pulsed surface dielectric barrier discharges for flow control. Plasma Physics and Controlled Fusion 52(12):124019 (2010).

38. Roupassov, D.V., Nikipelov, A.A., Nudnova, M.M., et al.: Flow separation control by plasma actuator with nanosecond pulsed-periodic discharge. AIAA journal 47(1): 168-185 (2009).

39. Zheng, J.G., Zhao, Z.J., Li, J., et al.: Numerical simulation of nanosecond pulsed dielectric barrier discharge actuator in a quiescent flow. Physics of Fluids. 26(3): 036102 (2014).

40. Komuro, A., Takashima, K., Tanaka, N., et al. Multiple control modes of nanosecond-pulse-driven plasma-actuator evaluated by forces, static pressure, and PIV measurements. Exp. Fluids 59(8), pp.129-1-19 (2018). 
41. Komuro, A., Takashima, K., Konno, K., et al. Schlieren visualization of flow-field modification over an airfoil by near-surface gas-density perturbations generated by a nanosecond-pulse-driven plasma actuator. J. Phys. D: Appl. Phys. 50(21), pp.215202-1-11 (2017).

42. Komuro, A., Matsuyuki, S., Ando,: Simulation of pulsed positive streamer discharges in air at high temperatures. Plasma Sources Sci. Technol. 27(10), pp 104005-1-18 (2018).

43. Brix „N Neuwerth, G., Jacob, D.: The inlet-vortex system of jet engines operating near the ground. 18th applied aerodynamics conference. 3998 (2000).

44. Seddon, and Goldsmith, E.L.: Intake Aerodynamics: an account of the mechanics of flow in and around the air intakes of turbine-engined and ramjet aircraft and missiles. Collins Professional and Technical Books, London. (1985).

45. Harrison, N.A., Anderson, J., Fleming, J.L., et al.: Active Flow Control of a Boundary Layer-Ingesting Serpentine Inlet Diffuser. Journal of Aircraft 50(1):262-271 (2013).

46. Colin, Y., Aupoix, B., Boussuge, J.F., et al.: Numerical simulation of the distortion generated by crosswind inlet flows. International Symposium on Air Breathing Engines (2007).

47. Greenblatt, D., Kastantin, Y., Nayeri, C.N., et al.: Delta-Wing Flow Control Using Dielectric Barrier Discharge Actuators. AIAA Journal 46(6):1554-1560 (2008).

48. Gursul, Wang, Z., Vardaki, E.: Review of flow control mechanisms of leading-edge vortices. Progress in Aerospace Sciences. 43(7-8): 246-270 (2007).

49. Jolibois, J., Forte, M., Moreau, É.: Application of an AC barrier discharge actuator to control airflow separation above a NACA 0015 airfoil: Optimization of the actuation location along the chord. Journal of Electrostatics 66(9-10): 496-503 (2008).

50. Porter, C., McLaughlin, T., Enloe, C., et al.: Boundary layer control using a DBD plasma actuator. 45th AIAA Aerospace Sciences Meeting and Exhibit. 786 (2007).

\section{Figures}




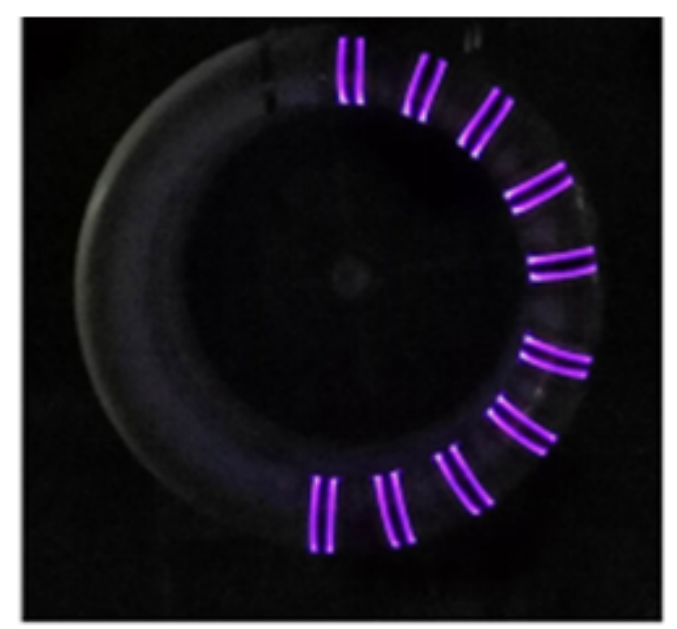

Streamwise

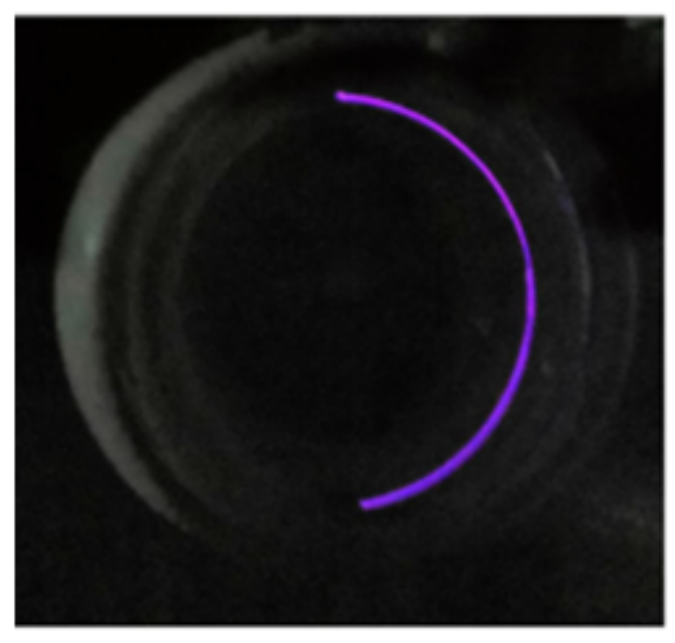

Circumferential

(a)

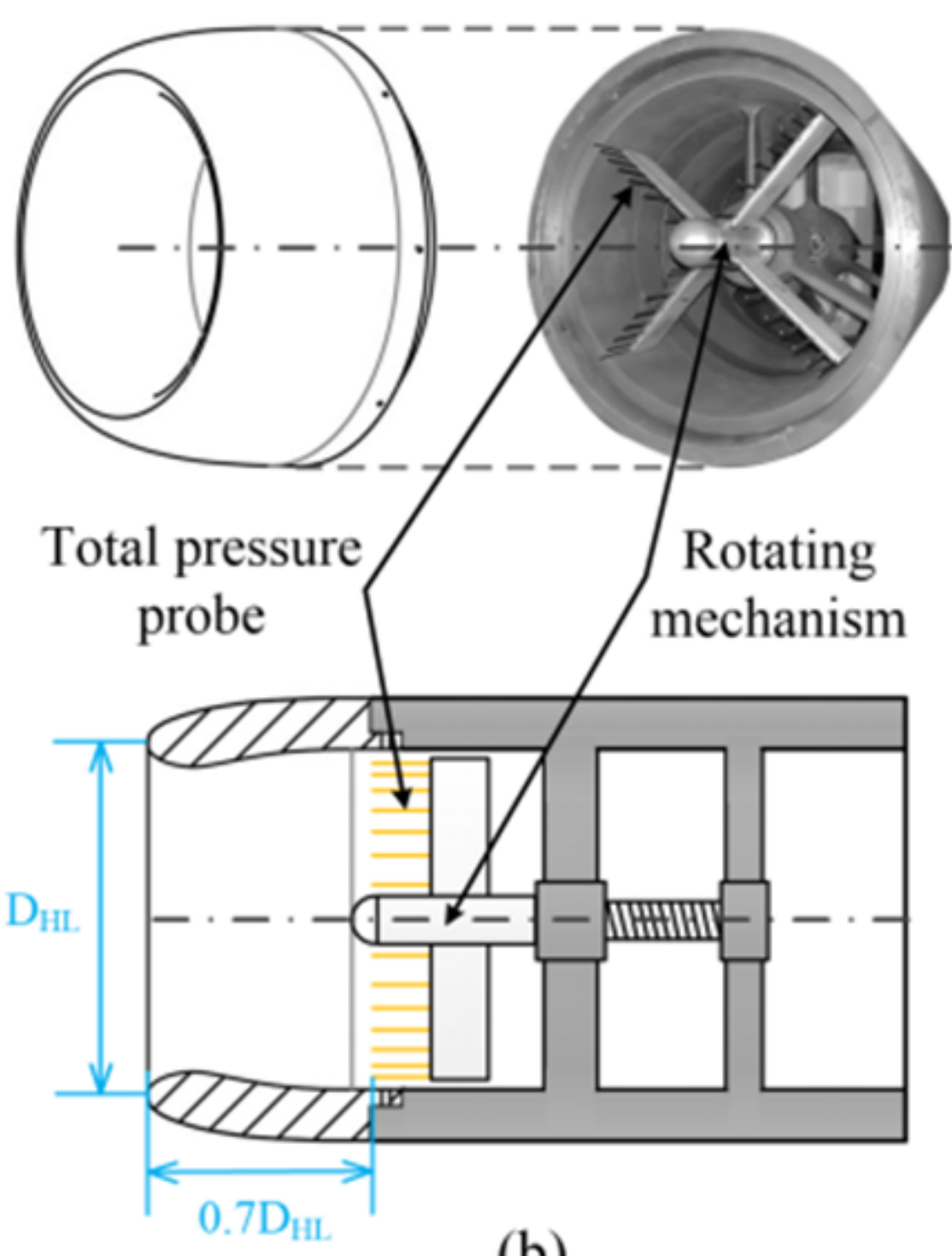

(b)

Figure 1

Structure of DLR-F6 nacelle scale model and experimental device 


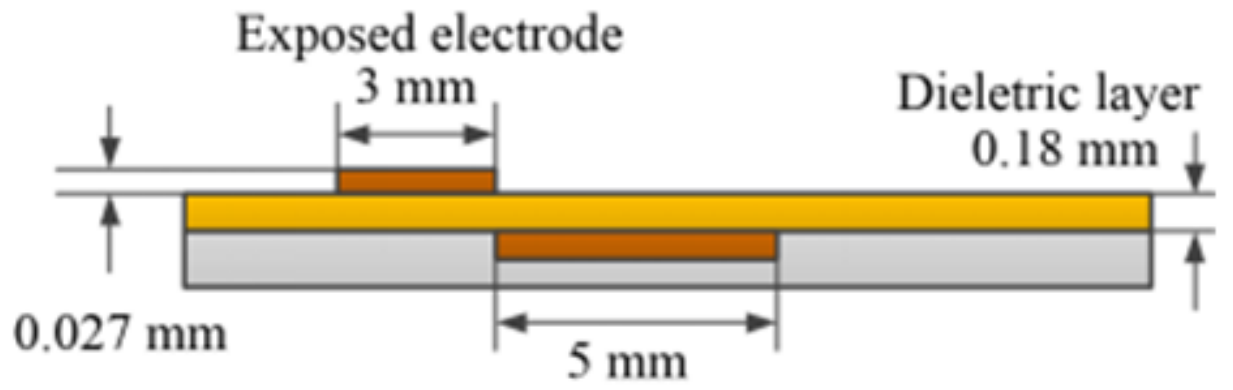

Covered electrode

(a)

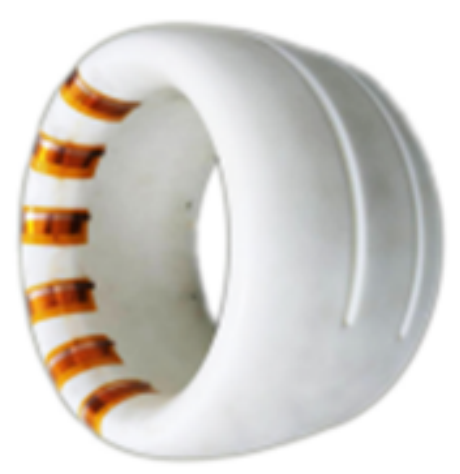

Streamwise

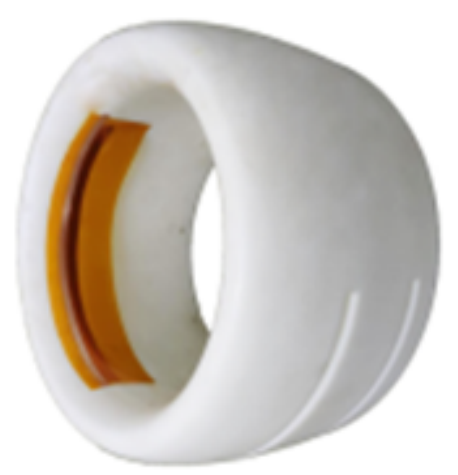

Circumferential

(b)

Figure 2

The plasma actuator and its installation in the nacelle 


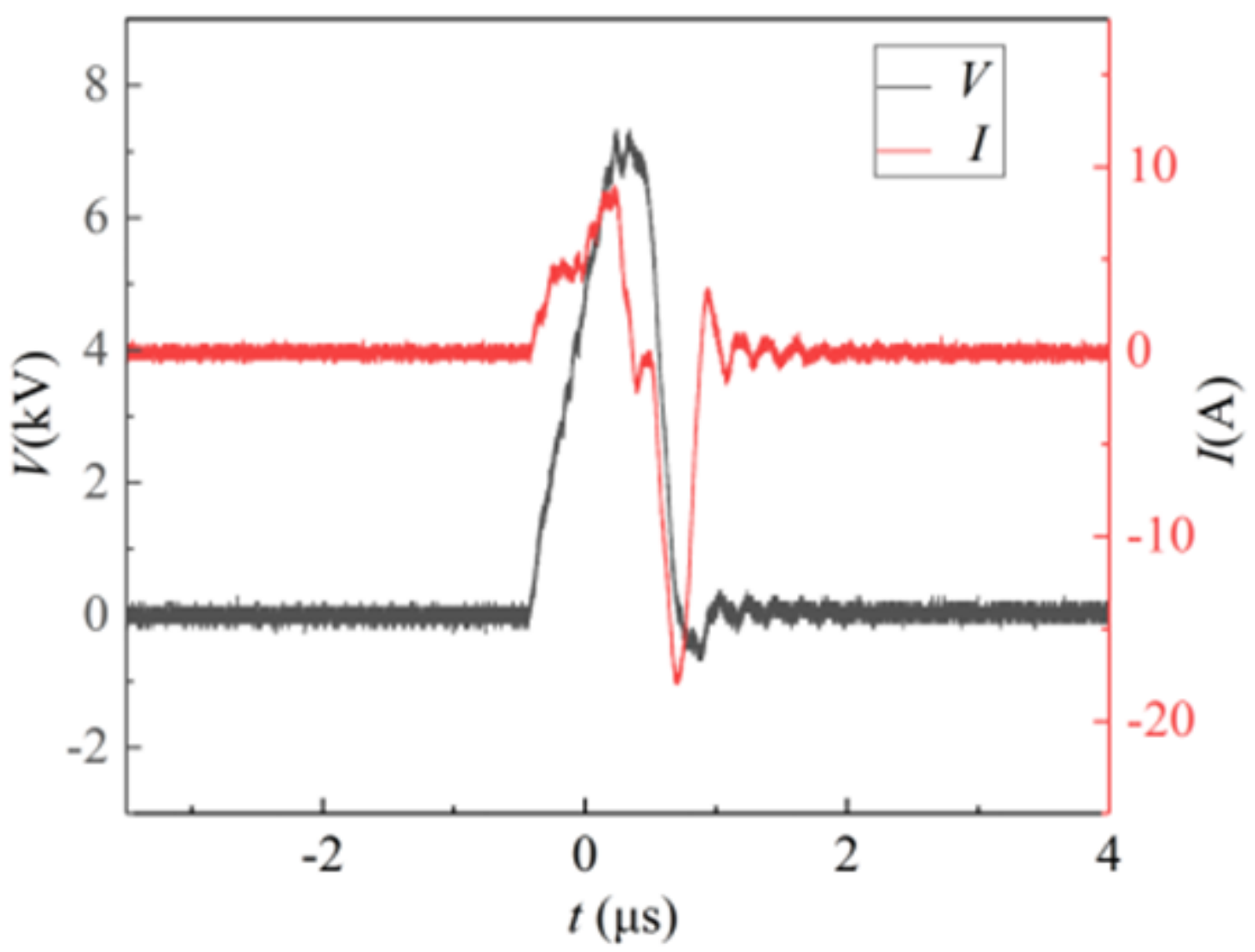

Figure 3

The voltage pulse and current profile of $\mu$ SDBD discharge

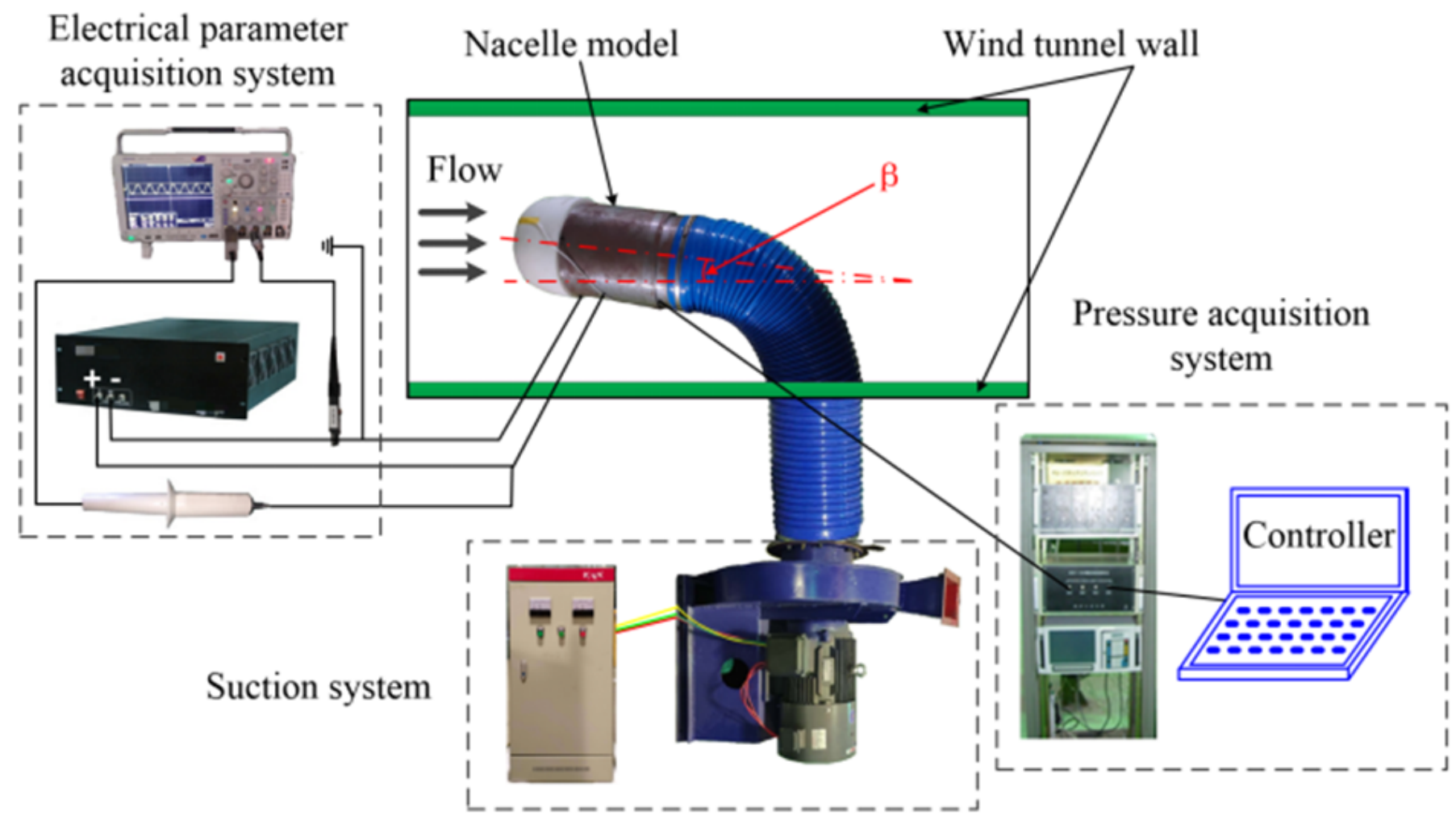


Figure 4

Integrated wind tunnel experiment system with airflow suction and data acquisition.

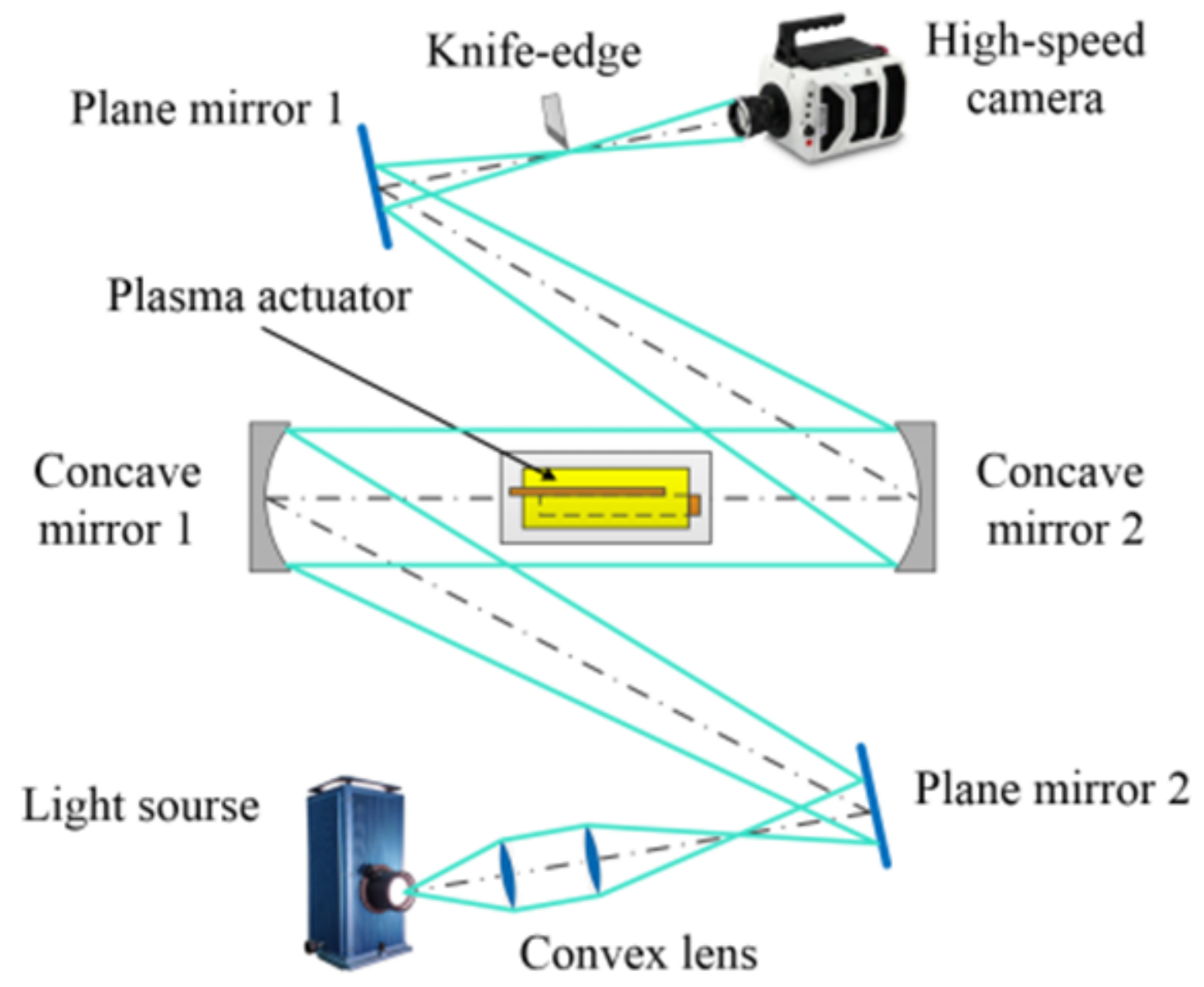

Figure 5

Schematic diagram of the schlieren system 

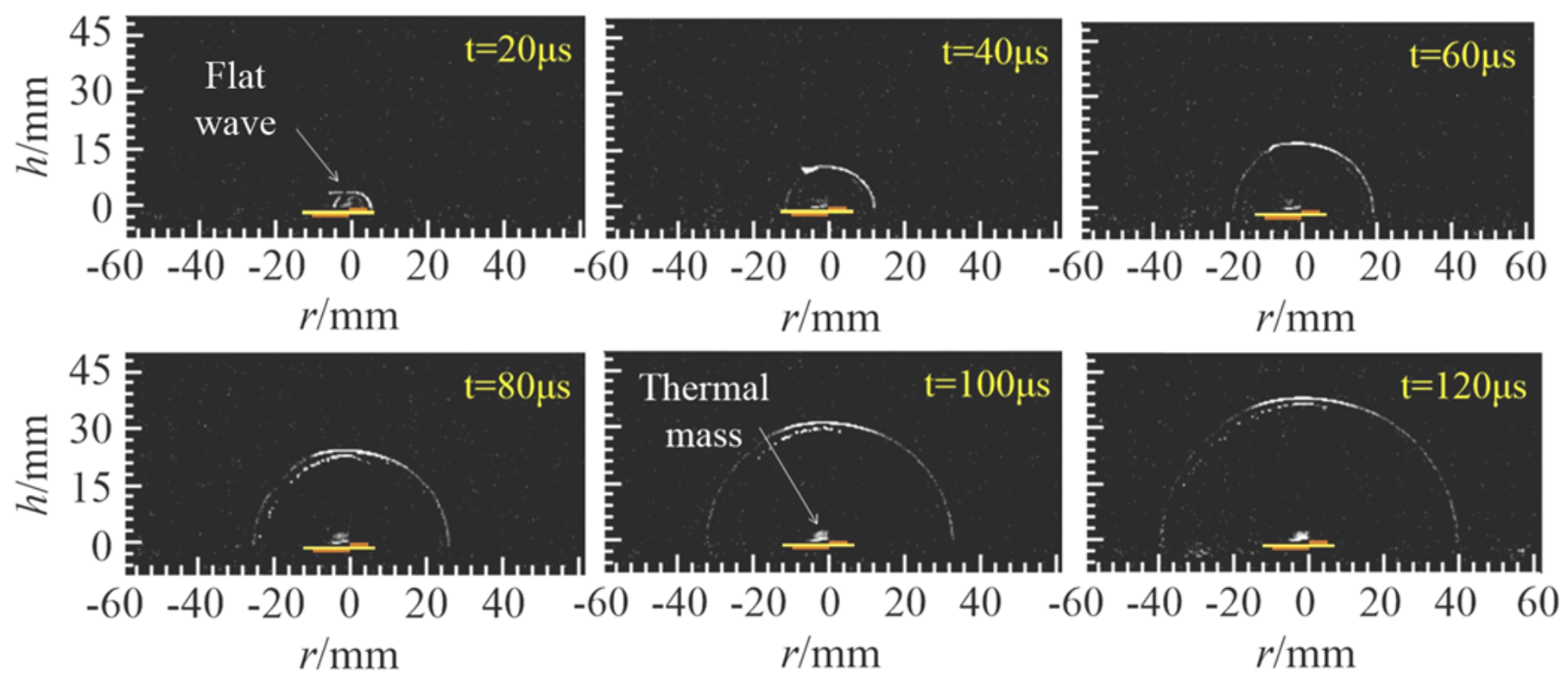

Figure 6

Pressure waves induced by $\mu$ SDBD (background normalization processing)

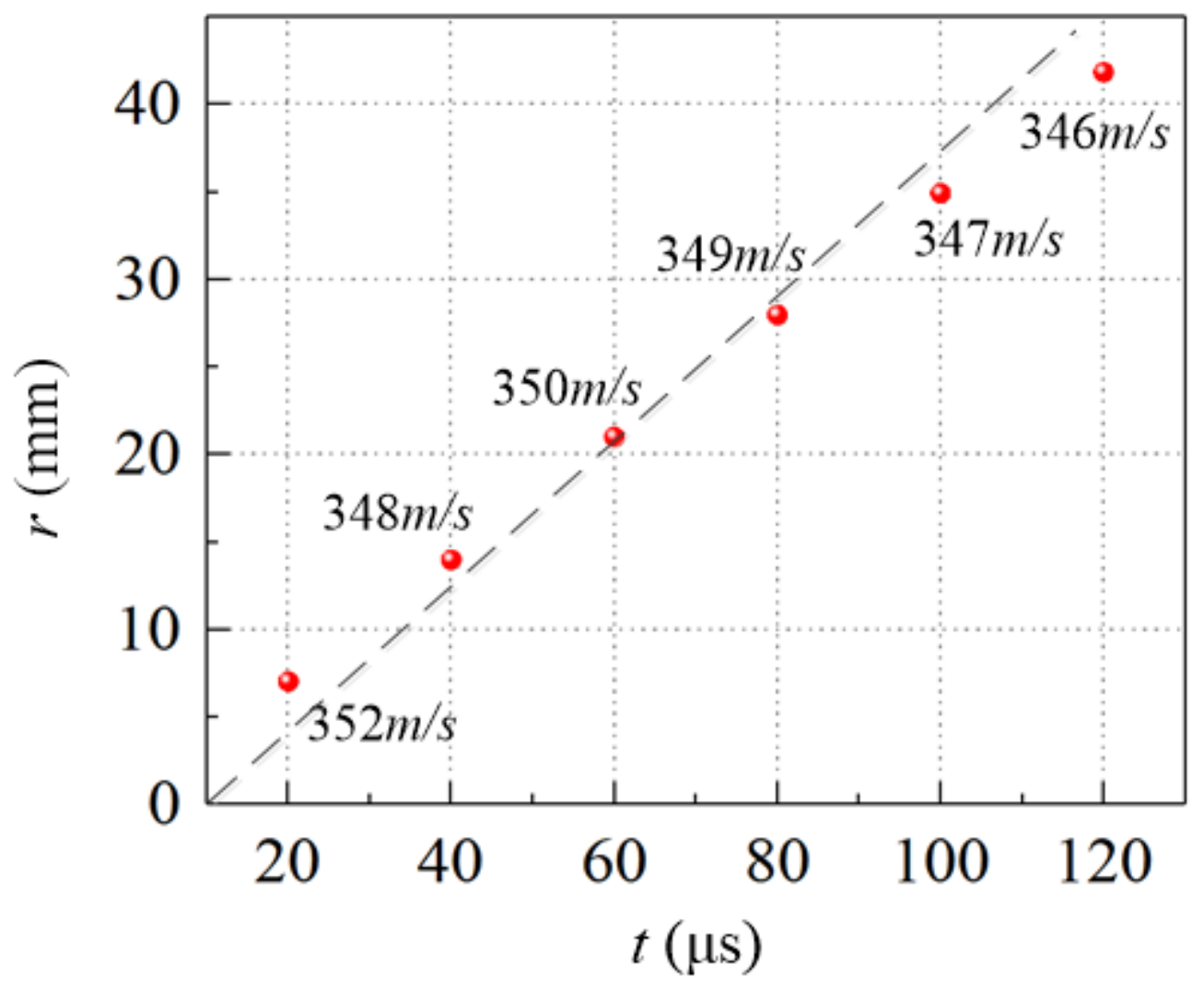

Figure 7

Radius and velocity of the pressure waves generated by $\mu$ SDBD actuation 


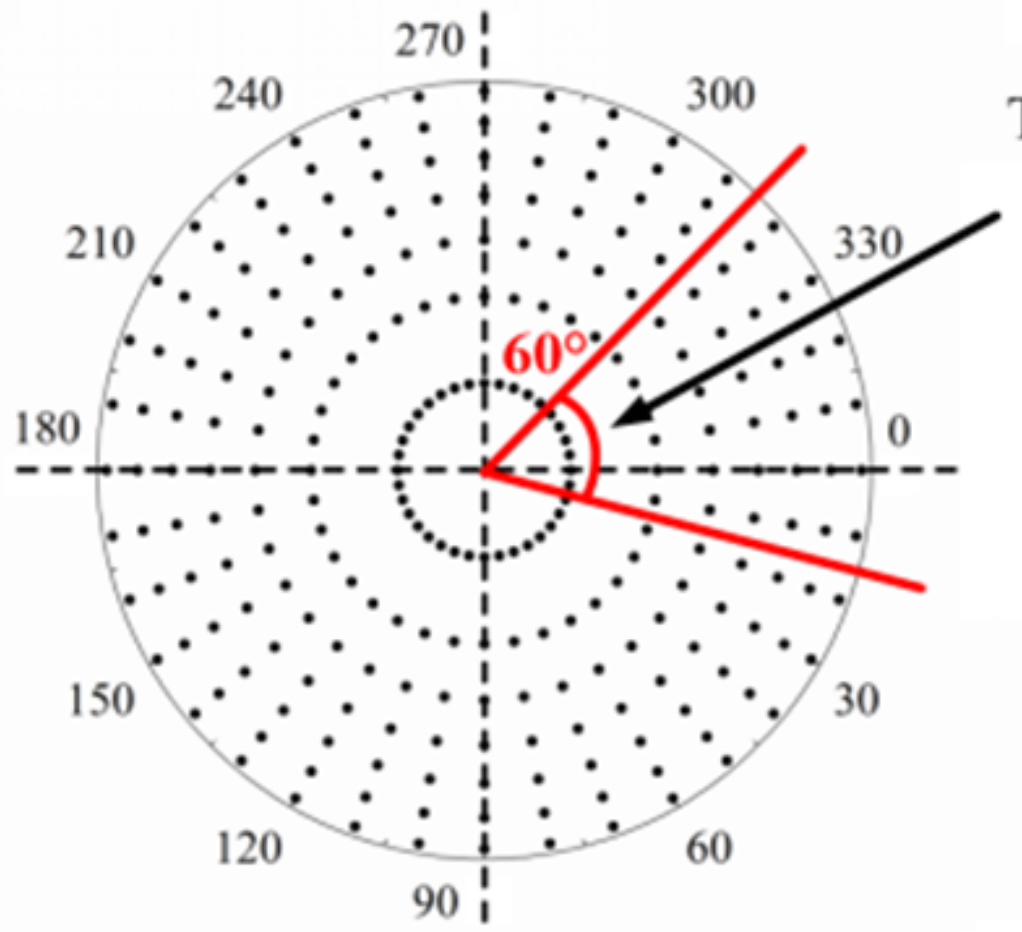

\section{The worst average} 60 degree sector

Figure 8

Schematic diagram of the worst average 60 degree sector

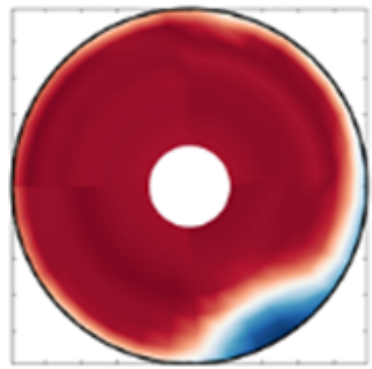

$\beta=10^{\circ}$

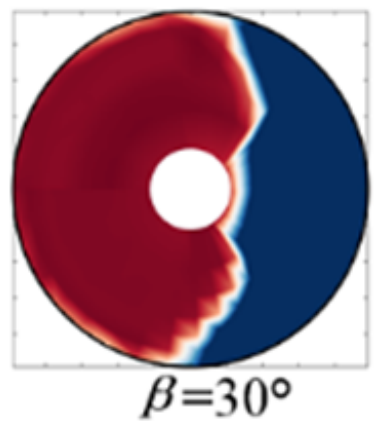

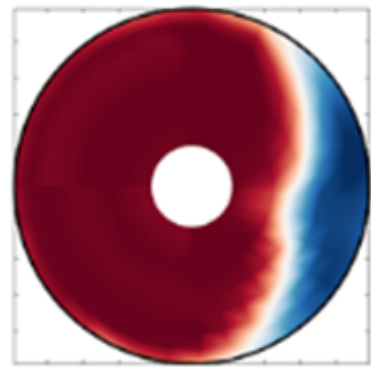

$\beta=14^{\circ}$

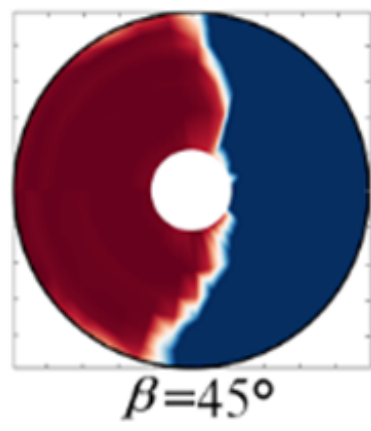

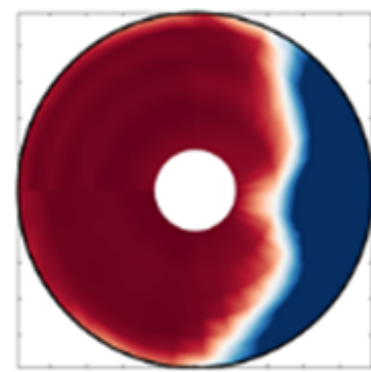

$\beta=18^{\circ}$

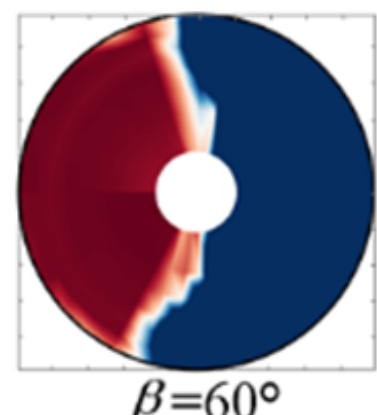

$\beta=60^{\circ}$

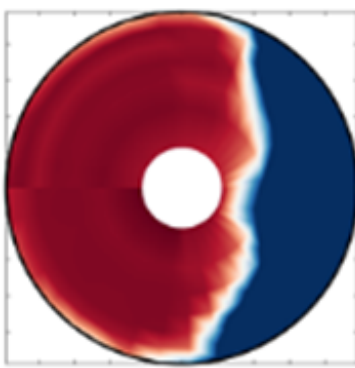

$\beta=22^{\circ}$

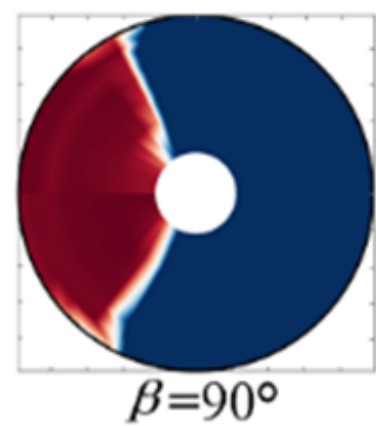

Figure 9

Distribution of the PR at air-intake exit under $\beta=10^{\circ} \sim 90^{\circ}$ (The crosswind comes from the right 


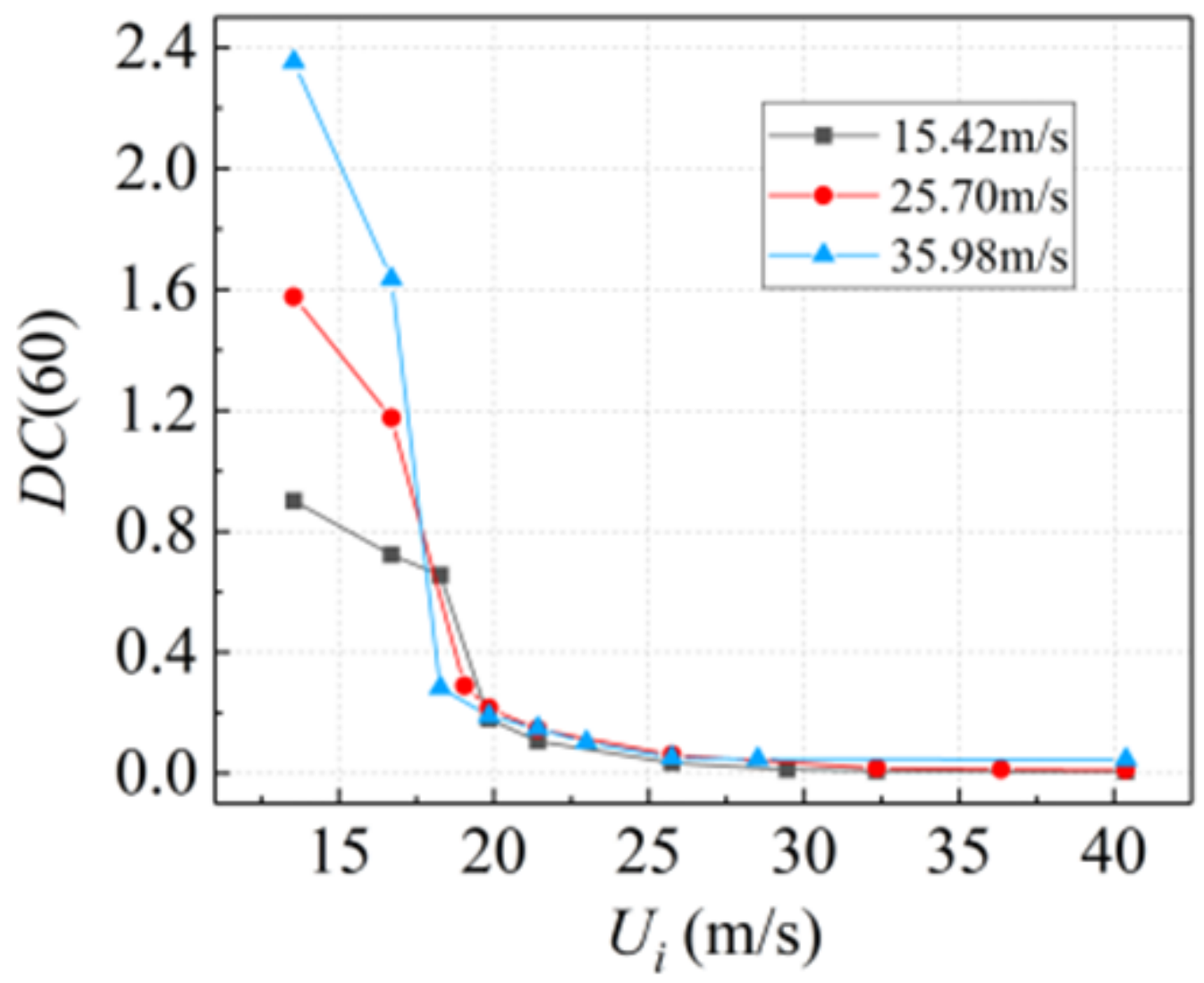

Figure 10

$D C(60)$ as the functions of $U_{i}$ at $U_{\infty}=15.42,25.70$ and $35.98 \mathrm{~m} / \mathrm{s}$ when $\beta=14^{\circ}$

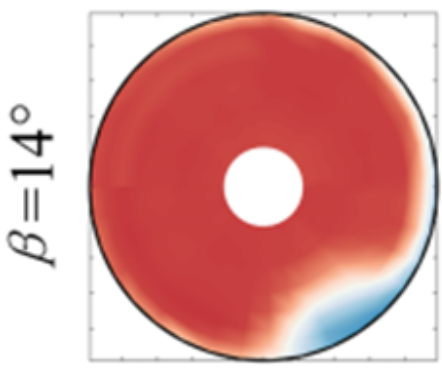

(a) Baseline

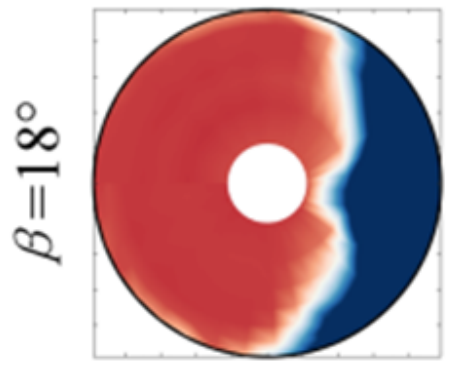

(e) Baseline

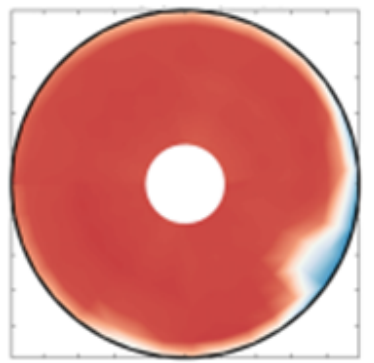

(b) $f=500 \mathrm{~Hz}$

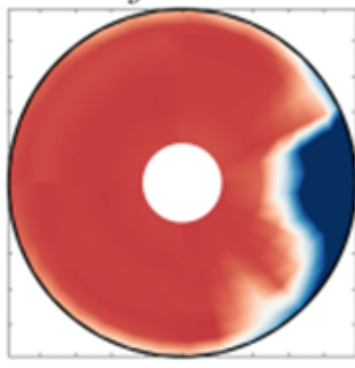

(f) $f=500 \mathrm{~Hz}$
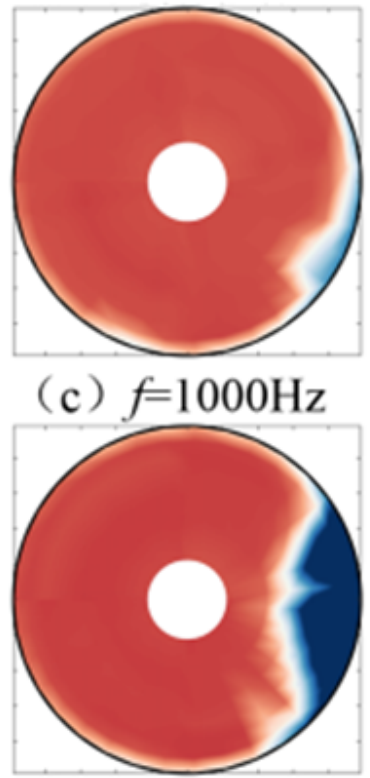

(g) $f=1000 \mathrm{~Hz}$
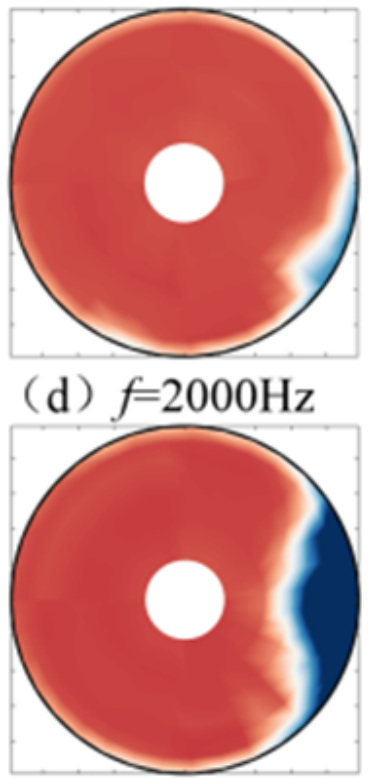

(h) $f=2000 \mathrm{~Hz}$

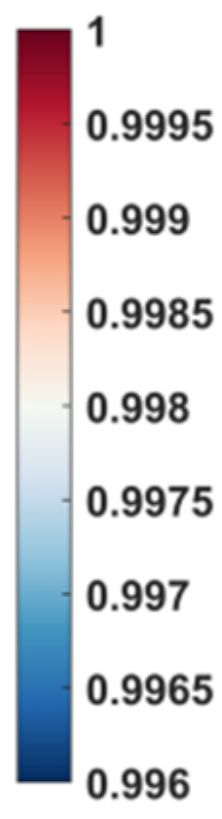

0.996

Figure 11 
Distribution of the PR of the air-intake exit in different actuation frequencies at $U_{\infty}=25.70 \mathrm{~m} / \mathrm{s}$ and $\mathrm{Ui}=$ $25.74 \mathrm{~m} / \mathrm{s}$

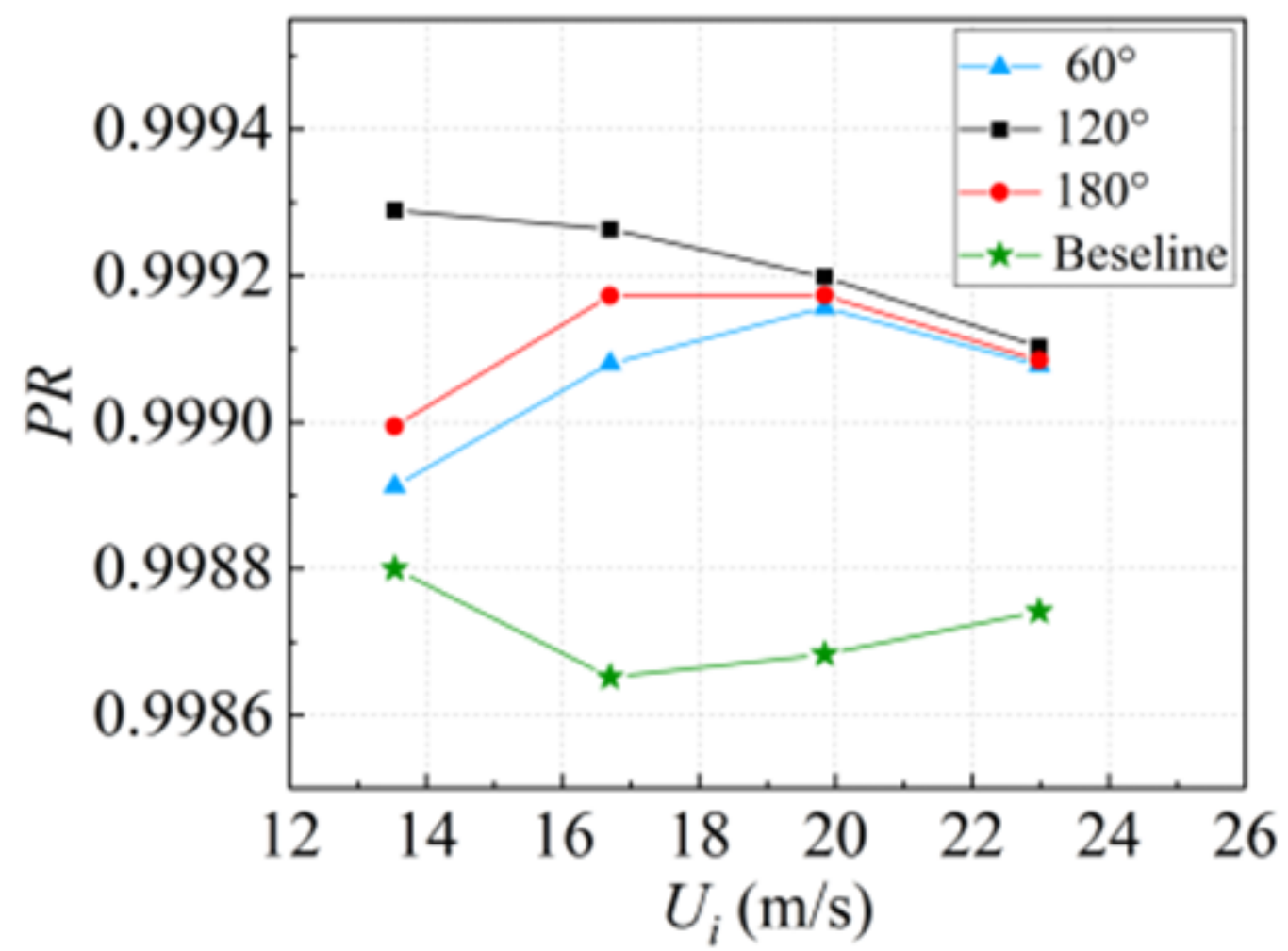

Figure 12

PR of the total pressure at air-intake exit under different actuator coverage at $f=500 \mathrm{~Hz}$ 

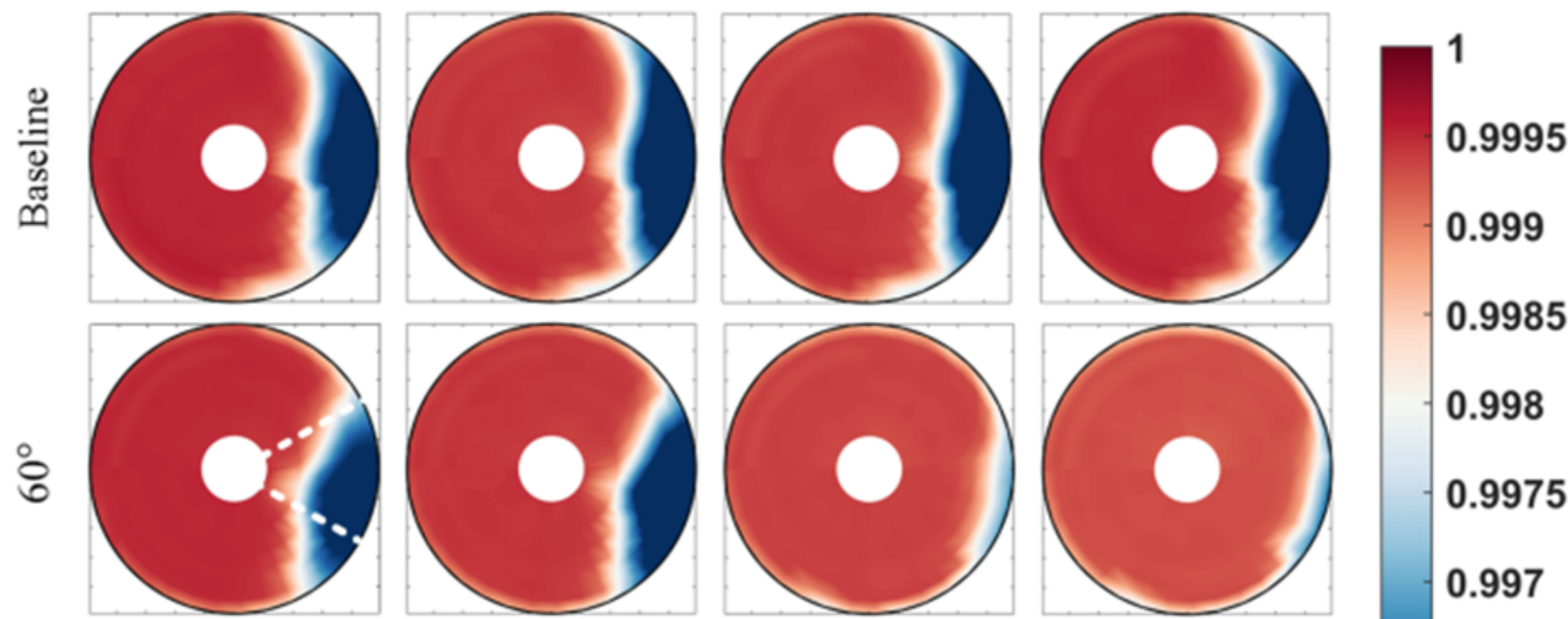

0.9985
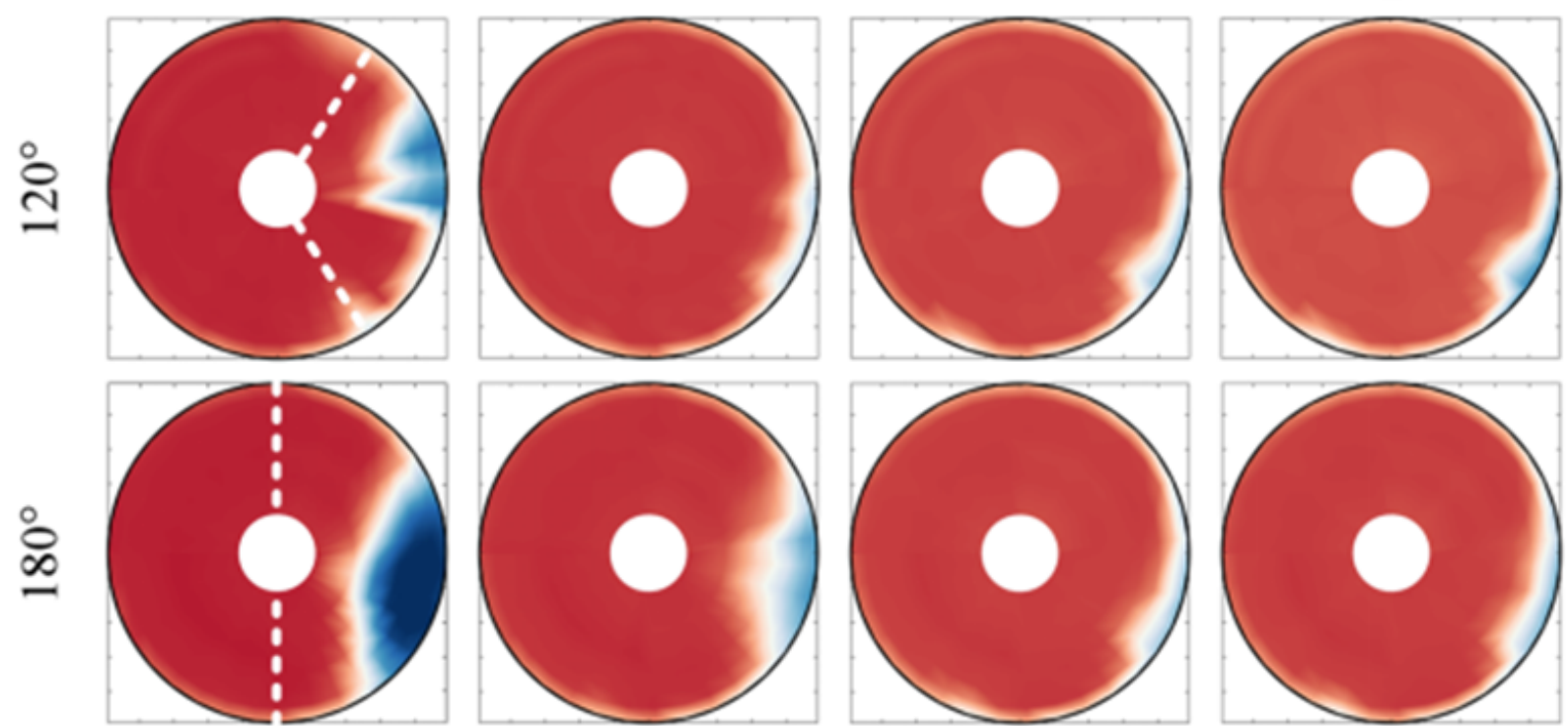

(a) $U_{i}=13.52 \mathrm{~m} / \mathrm{s}$ (b) $U_{i}=16.69 \mathrm{~m} / \mathrm{s}$

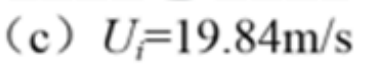

(d) $U_{i}=22.98 \mathrm{~m} / \mathrm{s}$

\section{Figure 13}

Distribution of the PR of the air-intake exit under different actuator coverage (Dotted lines indicate the beginning and end points of the coverage area)

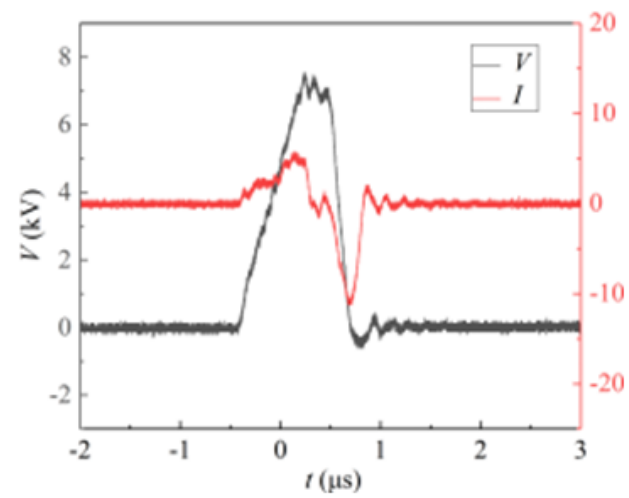

(a) $60^{\circ}$

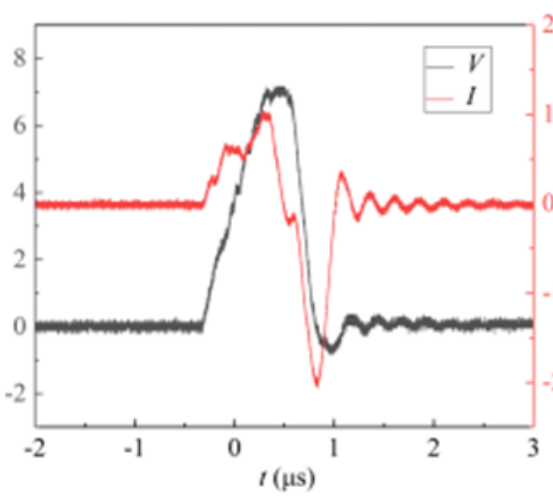

(b) $120^{\circ}$

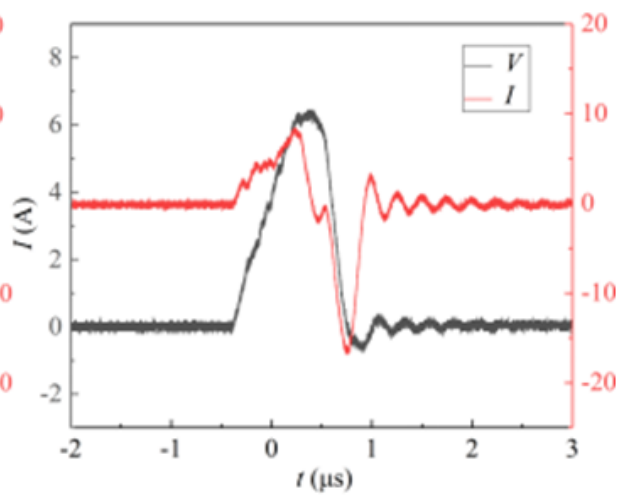

(c) $180^{\circ}$ 
Figure 14

The voltage pulse and current profile of $\mu$ SDBD discharge under different coverage area

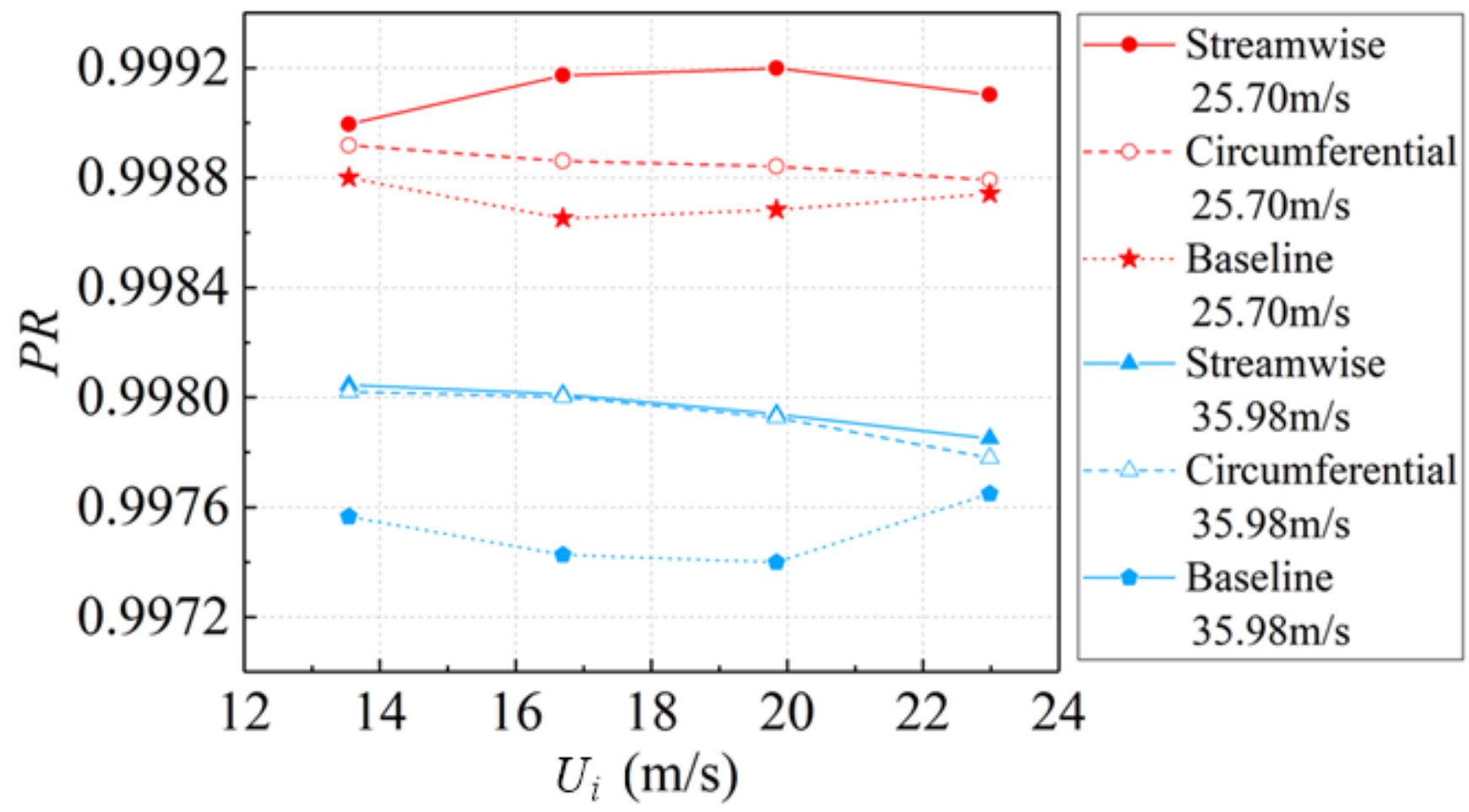

Figure 15

PR as the functions of Ui under different actuator layouts

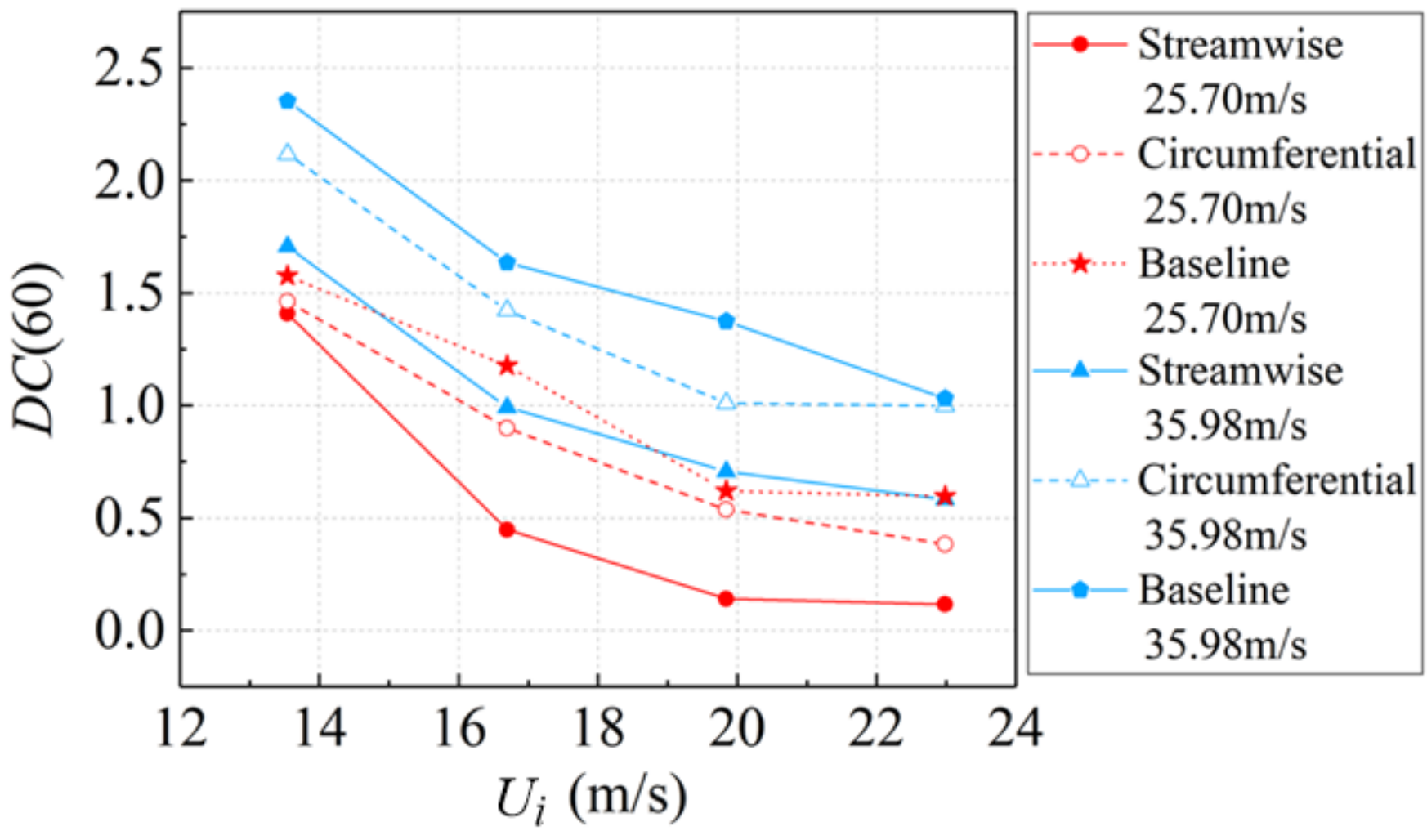


Figure 16

DC(60) as the functions of Ui under different actuator layouts
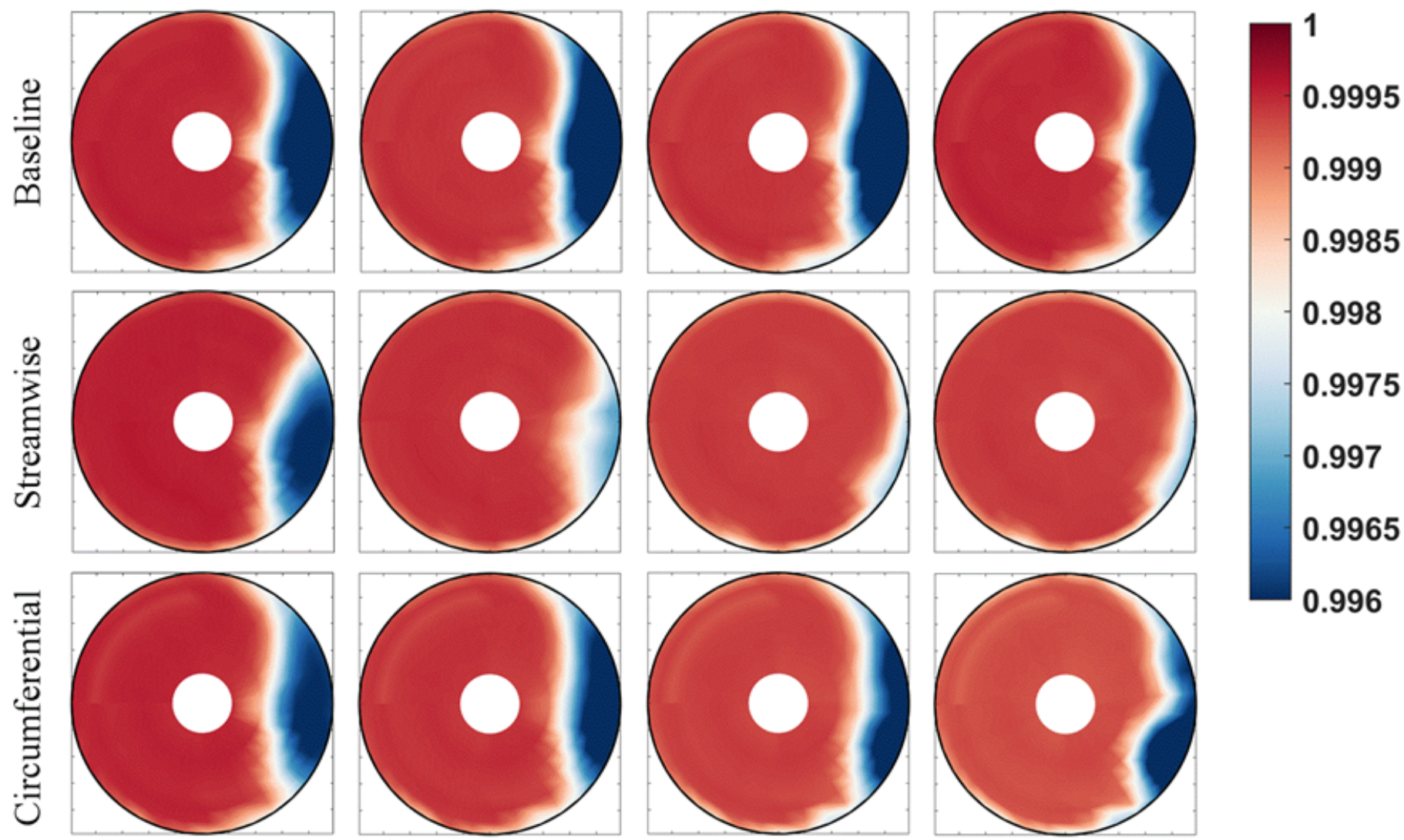

（a） $U_{i}=13.52 \mathrm{~m} / \mathrm{s}$ （b ） $U_{i}=16.69 \mathrm{~m} / \mathrm{s}$ （c） $U_{i}=19.84 \mathrm{~m} / \mathrm{s}$ （d） $U_{i}=22.98 \mathrm{~m} / \mathrm{s}$

Figure 17

Distribution of total pressure at air-intake exit under different actuator layouts at $U_{\infty}=25.70 \mathrm{~m} / \mathrm{s}$ 

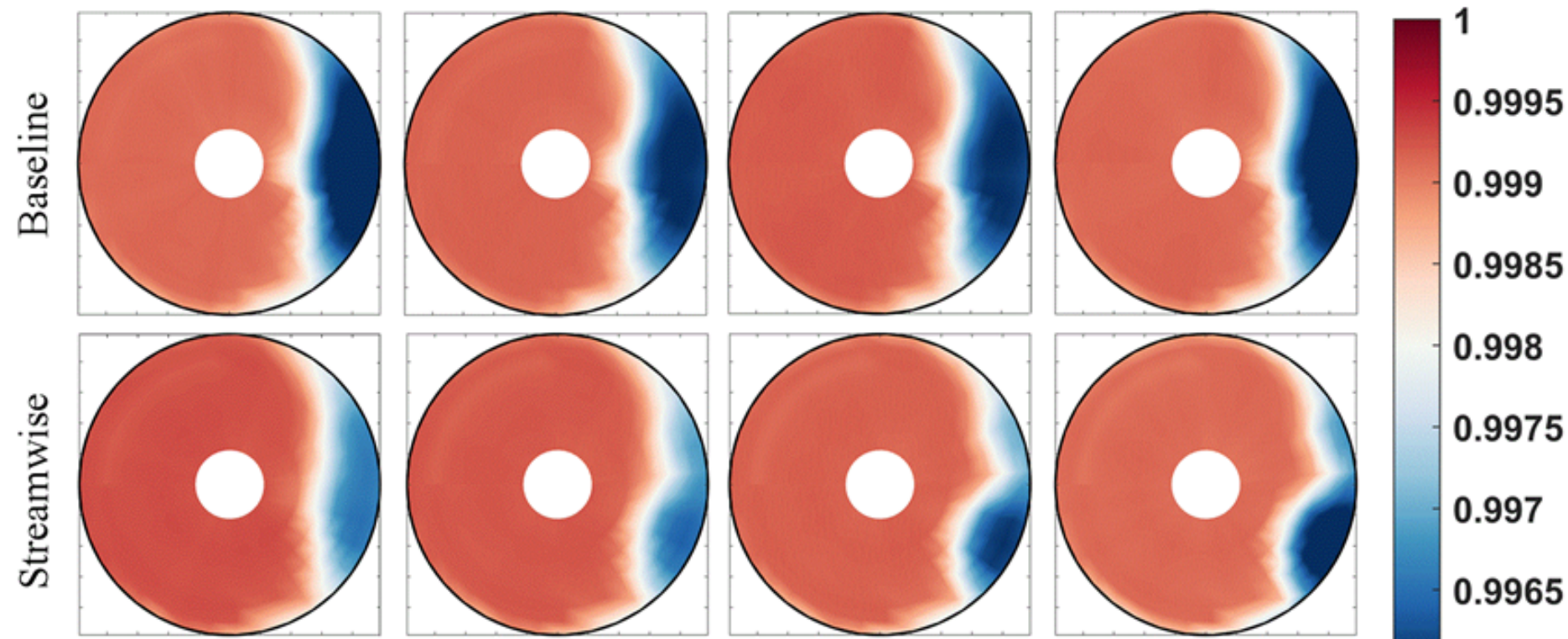

0.998
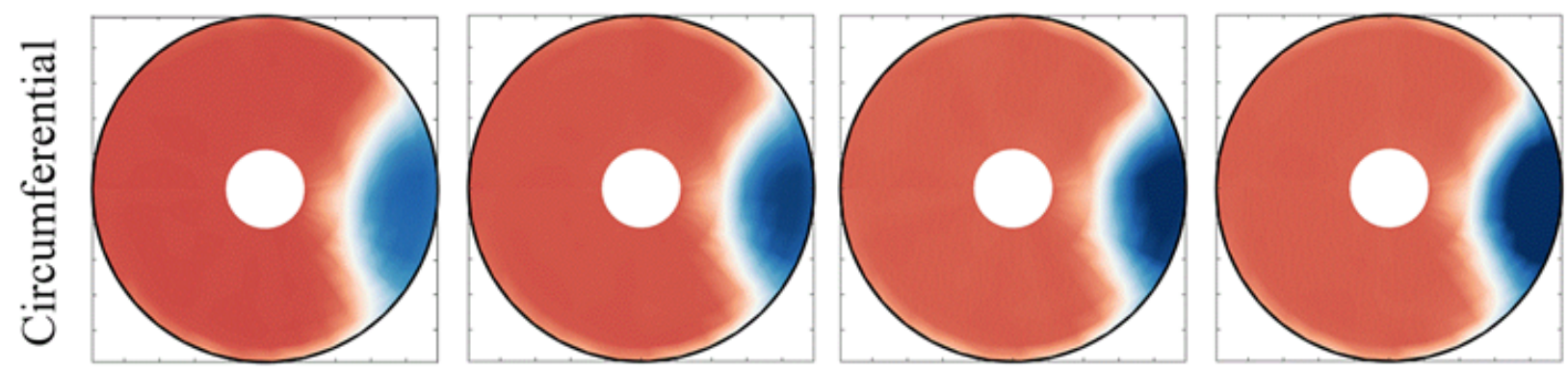

0.9975
0.997
0.9965
0.996

(a) $U_{i}=13.52 \mathrm{~m} / \mathrm{s}$
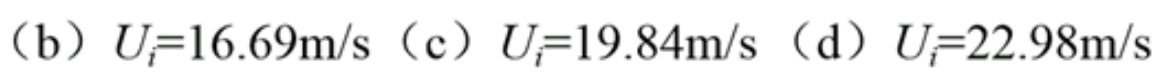

\section{Figure 18}

Distribution of total pressure at air-intake exit under different actuator layouts at $U_{\infty}=35.98 \mathrm{~m} / \mathrm{s}$ 